\title{
Twisted sheaves and the period-index problem
}

\author{
Max Lieblich
}

\begin{abstract}
We use twisted sheaves and their moduli spaces to study the Brauer group of a scheme. In particular, we (1) show how twisted methods can be efficiently used to re-prove the basic facts about the Brauer group and cohomological Brauer group (including Gabber's theorem that they coincide for a separated union of two affine schemes), (2) give a new proof of de Jong's period-index theorem for surfaces over algebraically closed fields, and (3) prove an analogous result for surfaces over finite fields. We also include a reduction of all period-index problems for Brauer groups of function fields over algebraically closed fields to characteristic zero, which (among other things) extends de Jong's result to include classes of period divisible by the characteristic of the base field. Finally, we use the theory developed here to give counterexamples to a standard type of local-to-global conjecture for geometrically rational varieties over the function field of the projective plane.
\end{abstract}

\section{Contents}

1 Introduction . . . . . . . . . . . . . . . . . . . . 1

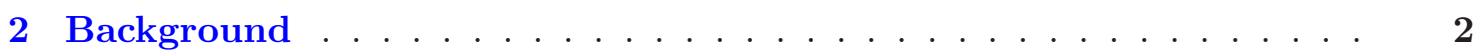

3 Twisted sheaves and their moduli . . . . . . . . . . . . . . . 5

4 Period-index results . . . . . . . . . . . . . . . . . . . . 19

Appendix A. A few facts about coherent sheaves on stacks . . . . . . 28

References . . . . . . . . . . . . . . . . . . . 29

\section{Introduction}

Let $K$ be a field. Given a Brauer class $\alpha \in \operatorname{Br}(K)$, there are two natural discrete invariants: the period of $\alpha, \operatorname{per}(\alpha)$, which is the order of $\alpha$ as an element of $\operatorname{Br}(K)$, and the index of $\alpha$, ind $(\alpha)$, whose square is the $K$-vector space dimension of a central division algebra with Brauer class $\alpha$. It is elementary to prove that $\operatorname{per}(\alpha) \mid \operatorname{ind}(\alpha)$ and that they have the same prime factors, so that $\operatorname{ind}(\alpha) \mid \operatorname{per}(\alpha)^{\ell}$ for some $\ell$ (see Lemma 2.1.1.3). The properties of the exponent $\ell$ have been studied by numerous authors (see [Art82], [Col02], [Dej04], [GY98], [Kre03], [Nak35], [Sal97, Sal98], [Van86], etc.), and the work to date has all supported the following folk-conjecture.

Conjecture. If $K$ is a $C_{d}$ field and $\alpha \in \operatorname{Br}(K)$, then $\operatorname{ind}(\alpha) \mid \operatorname{per}(\alpha)^{d-1}$.

The main goal of this paper is to prove the following theorem.

Theorem. Suppose that $K$ is a field of characteristic $p>0$ with transcendence degree two over the prime field. If $\alpha \in \operatorname{Br}(K)$ has order prime to $\operatorname{char}(K)$, then $\operatorname{ind}(\alpha) \mid \operatorname{per}(\alpha)^{3}$. If $\alpha$ is unramified, then $\operatorname{ind}(\alpha)=\operatorname{per}(\alpha)$.

Received 15 November 2005, accepted in final form 19 May 2007, published online 23 January 2008.

2000 Mathematics Subject Classification 14D20, 16K50.

Keywords: twisted sheaf, gerbe, period-index problem, Brauer group.

The author was partially supported by a Clay Liftoff Fellowship and an NSF Postdoctoral Fellowship during the preparation of this paper.

This journal is (C) Foundation Compositio Mathematica 2008. 


\section{LIEBLICH}

The reader will note that the exponent we obtain in the ramified case should be two according to the conjecture (although the unramified result is somewhat better than predicted). This gap is close to being filled, at the expense of adding another layer of stack-theoretic complexity to the methods employed here.

The class $\alpha$ is unramified precisely when it comes from a class in the Brauer group of a smooth projective model $X$ for the function field $K$. As we describe below, techniques of Saltman [Sal97, Sal98] permit the deduction of the ramified case from the unramified case. (It is precisely here where one is forced to increase the exponent by two rather than by one.) The unramified case is proven by finding a point in a certain moduli space parametrizing solutions to the equation per $=$ ind. We must of course first show that this moduli space is non-empty, which is precisely de Jong's periodindex theorem [Dej04]. We offer a new proof of this result, as well as a reduction of all period-index problems for function fields over algebraically closed fields to characteristic zero, thus giving an incremental improvement of de Jong's theorem: we do not need the hypothesis that the period of $\alpha$ is prime to the characteristic of the base field (until we start working over finite base fields).

The moduli space we use to prove these theorems is the space of stable twisted sheaves. As this paper is intended to encourage the use of twisted methods in the study of the Brauer group, we have included more than is strictly necessary for our purposes. We give a slightly different proof of Gabber's theorem that the Brauer group and cohomological Brauer group of an affine scheme coincide. (For an introduction to this problem, as well as a proof of Gabber's theorem of which ours is an adaptation, the reader is referred to [Hoo82].) The methods used here are a precursor to de Jong's proof of Gabber's more recent theorem: $\mathrm{Br}=\mathrm{Br}^{\prime}$ for any quasi-compact separated scheme admitting an ample invertible sheaf [Dej03].

The philosophy of twisted sheaves leads naturally to a question (Question 4.2.3.1) about the geometry of the moduli space of stable vector bundles with fixed determinant on a curve, whose answer is closely related to the period-index problem (in any dimension). By relating these two questions, we are able to give numerous examples of smooth projective geometrically connected geometrically rational varieties with geometric Picard group $\mathbb{Z}$ over $\mathbb{C}\left(t_{1}, t_{2}\right)$ which have points over the completion of $\mathbb{C}\left(t_{1}, t_{2}\right)$ at any (arbitrary) valuation but which lack rational points. These appear to be the first examples of such varieties over $\mathbb{C}\left(t_{1}, t_{2}\right)$ which violate the Hasse principle. (Hassett has informed me that there are also unpublished examples of cubic surfaces over $\mathbb{C}\left(t_{1}, t_{2}\right)$ which violate the Hasse principle and have vanishing Brauer-Manin obstruction; these of course have higher geometric Picard number.) It seems that the method given here for producing such examples might be useful in other contexts.

We now give a brief summary of the contents of this paper. In $\S 2$ we sketch the basic aspects of the period-index problem and the theory of gerbes (après Giraud); the reader familiar with this material can skip this section, referring back for terminology as required. This is followed in $\S 3$ by a rapid tour of the theory of twisted sheaves, their use in proving the basic facts about the Brauer group of a scheme and Gabber's theorem, and a summary of the theory of their moduli on low-dimensional ambient varieties (fully developed in [Lie07b]). Finally, in $\S 4$ we apply the abstract theory to the period-index problem and prove the main theorem. We have also included a very short appendix discussing a few basic facts about (quasi-)coherent sheaves on stacks and elementary transforms.

\section{Background}

\subsection{Algebra}

In this section we rapidly summarize the basic facts concerning the period-index problem. 


\section{TWISTED SHEAVES AND THE PERIOD-INDEX PROBLEM}

2.1.1 Period and index. The period-index problem has several manifestations. One way of describing the problem uses Galois cohomology. Let $K$ be a field and $F$ an étale sheaf on Spec $K$. Let $\alpha \in \mathrm{H}^{i}(\operatorname{Spec} K, F)$ be a Galois cohomology class with $i>0$.

Definition 2.1.1.1. The period of $\alpha$, denoted by $\operatorname{per}(\alpha)$, is the order of $\alpha$ in the group $\mathrm{H}^{i}(\operatorname{Spec} K, F)$. The (separable) index of $\alpha$, denoted by $\operatorname{ind}(\alpha)$, is $\operatorname{gcd}\left\{\operatorname{deg} L / K:\left.\alpha\right|_{L}=0\right\}$ with $L$ a separable extension.

In certain instances, the index of $\alpha$ is actually the minimal degree of a field extension killing $\alpha$ (for example, if $i=2$ and $F=\mathbf{G}_{m}$ ).

Example 2.1.1.2. When $F=\mathbf{G}_{m}$ and $i=2$, we can translate Definition 2.1.1.1 into the language of non-commutative algebra. A class $\alpha \in \mathrm{H}^{2}\left(\operatorname{Spec} K, \mathbf{G}_{m}\right)=\operatorname{Br}(K)$ is represented by a central division $K$-algebra $D$. The period of $\alpha$ is the order of $[D]$ in $\operatorname{Br}(K)$ and the index of $\alpha$ is the square root of the rank of $D$. This follows from basic theorems in non-commutative algebra [FD93] which describe the structure of central division algebras.

Lemma 2.1.1.3. Given $F, i$, and $\alpha$ as above, $\operatorname{per}(\alpha) \mid \operatorname{ind}(\alpha)$ and both have the same prime factors.

Proof. This is well-known, but we review the proof for the sake of completeness. That $\operatorname{per}(\alpha) \mid \operatorname{ind}(\alpha)$ is a consequence of the existence, for any finite separable extension $f: \operatorname{Spec} L \rightarrow \operatorname{Spec} K$, of a trace map $f_{*} f^{*} F \rightarrow F$ (the 'corestriction') such that the composition with the restriction is multiplication by $\operatorname{deg} L / K$. Applying this to $L / K$ such that $\alpha_{L}=0$ shows that the degree of any such extension kills $\alpha$, whence the gcd kills $\alpha$. To show that the prime factors are the same, suppose that $L / K$ kills $\alpha$ and let $p$ be a prime number not dividing $\operatorname{per}(\alpha)$. Suppose that $L$ is Galois with group $G$. Let $G_{p}$ be a $p$-Sylow subgroup of $G$ and $L_{p}$ the fixed field. Thus, the degree of $L_{p}$ over $K$ has no factors of $p$. We claim that $L_{p}$ kills $\alpha$. Indeed, $\alpha_{L_{p}}$ is still $\operatorname{per}(\alpha)$-torsion and $p$ still acts invertibly on the subgroup generated by $\alpha$. Thus, restricting further to $L$ and corestricting back to $L_{p}$, we see that $\alpha$ is trivial over $L_{p}$ if and only if it is trivial over $L$. This removes $p$ from $\operatorname{ind}(\alpha)$. The case of general separable $L / K$ follows from the case of Galois $L / K$.

Thus, there is some minimal $\ell_{\alpha}$ so that $\operatorname{ind}(\alpha) \mid \operatorname{per}(\alpha)^{\ell_{\alpha}}$. The period-index problem is to understand the behavior of the exponent $\ell_{\alpha}$ as $K$ and $\alpha$ vary. In the context of elliptic curves, this was considered by Lang-Tate [T58] and Lichtenbaum [Lic68]. Using Example 2.1.1.2, we see that the period-index problem for the Brauer group is related to deciding how large a division algebra is necessary to represent a given Brauer class. In this form, it was considered by Brauer in his foundational paper and by Albert in subsequent work.

Remark 2.1.1.4. Let $\pi: E \rightarrow \mathbf{P}^{1}$ be an elliptic surface over a finite field (with a section $\sigma$ ). It is a classical result [Sil92, Exercise 10.11] that given an element $\alpha \in \amalg\left(k\left(\mathbf{P}^{1}\right), E_{\eta}\right)$ of period $n$ prime to the characteristic of $k$, there is a field extension of degree $n$ of $k\left(\mathbf{P}^{1}\right)$ killing $\alpha$. In other words, for these particular classes of $\mathrm{H}^{1}\left(\operatorname{Spec} k\left(\mathbf{P}^{1}\right), E_{\eta}\right)$, the period equals the index. Using Artin's isomorphism $\operatorname{Br}(E) \stackrel{\sim}{\rightarrow} \amalg\left(k\left(\mathbf{P}^{1}\right), E_{\eta}\right)$ (see [Gro68b]), we see that this inadvertently also proves that $\operatorname{per}(\alpha)=\operatorname{ind}(\alpha)$ for any $\alpha \in \operatorname{Br}(E)$.

2.1.2 $\mathrm{Br}$ and $\mathrm{Br}^{\prime}$. The purpose of this section is primarily to fix notation. Given any ringed topos $X$, there is a natural injection $\operatorname{Br}(X) \hookrightarrow \mathrm{H}^{2}\left(X, \mathbf{G}_{m}\right)$ (developed using the language of gerbes in [Gir71, $\S$ V.4]). The torsion subgroup of $\mathrm{H}^{2}\left(X, \mathbf{G}_{m}\right)$ is the cohomological Brauer group, denoted by $\mathrm{Br}^{\prime}$.

Question 2.1.2.1. When is the natural map $\mathrm{Br} \rightarrow \mathrm{Br}^{\prime}$ an isomorphism? 


\section{LIEBLICH}

Grothendieck showed that $\mathrm{Br}=\mathrm{Br}^{\prime}$ for Noetherian schemes of dimension one and regular schemes of dimension two [Gro68a]. In his thesis [Gab81], Gabber showed that this is so for any scheme $X$ which can be written as a separated union of two affines. We give a proof of all of these results using twisted sheaves in $\S 3.1 .4$. A more recent result of Gabber (mid-1990s, unpublished) established that $\mathrm{Br}=\mathrm{Br}^{\prime}$ for any quasi-projective scheme, for which de Jong has given a twisted proof [Dej03]. There is an example of a non-separated normal surface $Z$ such that $\operatorname{Br}(Z)[2] \neq \operatorname{Br}^{\prime}(Z)[2]$ (see [EHKV01]). The question is still quite open, even for smooth non-quasiprojective threefolds over algebraically closed fields!

\subsection{Geometry}

We follow the terminology and conventions of [LM00] when discussing stacks and algebraic stacks. Given a stack $\mathscr{X}$ over an algebraic space $X$, we use $\mathscr{I}(\mathscr{X})$ to denote the inertia stack, a representable group-functor on $\mathscr{X}$ which associates to $\varphi: T \rightarrow \mathscr{X}$ the automorphisms of $\varphi$. (One can also explicitly describe the inertia stack as $\mathscr{I}(\mathscr{X})=\mathscr{X} \times \mathscr{X} \times \mathscr{X} \mathscr{X}$, with both maps being the diagonal morphism. It is then an exercise to check that this represents the sheaf of automorphisms, as described above. It appears that the inertia stack lacks a standard reference in the literature.) We write $\operatorname{Sh}(\mathscr{X})$ to denote the sheafification of $\mathscr{X}$, by which we mean the universal object among sheaves $\mathscr{F}$ on $X$ admitting a map of stacks $\mathscr{X} \rightarrow \mathscr{F}, \mathscr{F}$ being considered as a stack via the natural functor from sheaves to stacks.

2.2.1 Gerbes: generalities. The canonical reference for gerbes and non-abelian cohomology is [Gir71], especially Chapter IV. Fix a positive integer $n$.

Situation 2.2.1.1. For the sake of simplicity, we assume that $n$ is prime to the characteristics of $X$.

Definition 2.2.1.2. A gerbe on $X$ is a stack $\mathscr{X} \rightarrow X$ such that the sheafification $\operatorname{Sh}(\mathscr{X}) \rightarrow X$ is an isomorphism. This is equivalent to the following two conditions:

(i) for every open set $U \rightarrow X$, there is a covering $V \rightarrow U$ such that the fiber category $\mathscr{X}_{V} \neq \emptyset$;

(ii) given an open set $U \rightarrow X$ and any two objects $x, y \in \mathscr{X}_{U}$, there is a covering $V \rightarrow U$ and an isomorphism $x_{V} \stackrel{\sim}{\rightarrow} y_{V}$.

Definition 2.2.1.3. Given an abelian sheaf $A$ on $X$, an $A$-gerbe on $X$ is a gerbe $\mathscr{X}$ along with an isomorphism $A_{\mathscr{X}} \stackrel{\sim}{\rightarrow} \mathscr{I}(\mathscr{X})$. An isomorphism of $A$-gerbes $\mathscr{X}$ and $\mathscr{Y}$ is a 1 -morphism $f: \mathscr{X} \rightarrow \mathscr{Y}$ which is a 1-isomorphism of stacks such that the natural map

$$
A_{\mathscr{X}} \stackrel{\sim}{\rightarrow} \mathscr{I}(\mathscr{X}) \rightarrow f^{*} \mathscr{I}(\mathscr{Y}) \stackrel{\sim}{\rightarrow} A_{\mathscr{X}}
$$

is the identity.

The primordial example of an $A$-gerbe is the classifying stack B $A$ of $A$-torsors. A general $A$-gerbe should then be thought of as a bundle with fiber $\mathrm{B} A$ and ' $A$-linear' gluing data. The original interest in these objects comes from the following result of Giraud. For more on this subject, the reader is of course referred to [Gir71, §IV.3], and may consult [Lie04] for a more condensed discussion.

Proposition 2.2.1.4 (Giraud). There is a natural bijection between the set of isomorphism classes of $A$-gerbes and $\mathrm{H}^{2}(X, A)$.

Notation 2.2.1.5. We write $[\mathscr{X}]$ for the class of $\mathscr{X}$ in $\mathrm{H}^{2}(X, A)$.

Recall that given a sheaf $\mathscr{F}$ on a stack $\mathscr{X}$, there is a natural right action $\mathscr{F} \times \mathscr{I}(\mathscr{X}) \rightarrow \mathscr{F}$. Indeed, given an object $U$ in the site of $\mathscr{X}$ and a section $(f, \sigma)$ of $\mathscr{F} \times \mathscr{I}(\mathscr{X})$ over $U$, we have an isomorphism $\sigma^{*}: \mathscr{F}(U) \stackrel{\sim}{\rightarrow} \mathscr{F}(U)$, and we let $(f, \sigma)$ map to $\sigma^{*} f$. On an $A$-gerbe, this becomes an action $\mathscr{F} \times A_{\mathscr{X}} \rightarrow \mathscr{F}$. 


\section{TWISTED SHEAVES AND THE PERIOD-INDEX PROBLEM}

Definition 2.2.1.6. Given an $A$-gerbe $\mathscr{X}$ and a sheaf on $\mathscr{X}$, we call the natural action $\mathscr{F} \times A \rightarrow \mathscr{F}$ the inertial action.

2.2.2 Gerbes: two special flavors. We briefly describe two different types of $\boldsymbol{\mu}_{n}$-gerbes which are important for us. Throughout, $X$ is assumed to be a $k$-scheme for some field $k$ such that $n \in k^{\times}$. (This last requirement is not necessary if one is willing to work in the flat topology.)

Definition 2.2.2.1. A $\boldsymbol{\mu}_{n}$-gerbe $\mathscr{X} \rightarrow X$ is (geometrically) essentially trivial if the class [ $\mathscr{X}$ ] has trivial image in $\mathrm{H}^{2}\left(X, \mathbf{G}_{m}\right)$ (respectively, $\mathrm{H}^{2}\left(X \otimes_{k} \bar{k}, \mathbf{G}_{m}\right)$ ).

Definition 2.2.2.2. Let $\mathscr{M}$ be an invertible sheaf on $X$. The gerbe of $n$th roots of $\mathscr{M}$, denoted by $[\mathscr{M}]^{1 / n}$, is the stack whose objects over $T$ are pairs $(\mathscr{L}, \varphi)$, where $\mathscr{L}$ is an invertible sheaf on $X \times T$ and $\varphi: \mathscr{L}^{\otimes n} \stackrel{\sim}{\rightarrow} \mathscr{M}$ is an isomorphism.

It is immediate that $[\mathscr{M}]^{1 / n}$ is a $\boldsymbol{\mu}_{n}$-gerbe. A consideration of the Kummer sequence $1 \rightarrow \boldsymbol{\mu}_{n} \rightarrow$ $\mathbf{G}_{m} \rightarrow \mathbf{G}_{m} \rightarrow 1$ shows that every essentially trivial $\boldsymbol{\mu}_{n}$-gerbe is the gerbe of $n$th roots of some invertible sheaf. Geometrically essentially trivial gerbes are more difficult to describe.

On the opposite side of the essentially trivial gerbes are the optimal gerbes.

Definition 2.2.2.3. A $\boldsymbol{\mu}_{n}$-gerbe $\mathscr{X} \rightarrow X$ is (geometrically) optimal if the associated $\mathbf{G}_{m}$-gerbe has order $n$ in $\mathrm{H}^{2}\left(X, \mathbf{G}_{m}\right)$ (respectively, in $\mathrm{H}^{2}\left(X \otimes \bar{k}, \mathbf{G}_{m}\right)$ ).

If $X$ is regular, this is the same as saying that the generic fiber of $\mathscr{X} \rightarrow X$ has order $n$ in $\mathrm{H}^{2}\left(\eta_{X}, \boldsymbol{\mu}_{n}\right)$ (respectively, in $\mathrm{H}^{2}\left(\eta_{X \otimes \bar{k}}, \boldsymbol{\mu}_{n}\right)$ ), which is the same as saying that $\mathscr{X}_{\eta}$ is the $\boldsymbol{\mu}_{n}$-gerbe associated to a division algebra over $\kappa(\eta)$ of rank $n^{2}$. If there is an Azumaya algebra of degree $n$ on $X$ in the class $[\mathscr{X}]$, this is perhaps saying that 'Spec $\mathscr{A}$ is an integral non-commutative surface finite, flat, and unramified over its center'.

\section{Twisted sheaves and their moduli}

In this section we introduce the category of twisted sheaves and show its suitability for studying certain types of questions about the Brauer group. We also give a summary of the results of [Lie07b], which studies the properties of the moduli spaces of twisted sheaves.

\subsection{Twisted sheaves}

Let $X$ be an algebraic space. We equip $X$ with a reasonable flat topology: the big or small étale topology or the fppf topology. When we speak of a stack on $X$, we mean a stack in this topology. When studying sheaves on a stack, we use the big étale or fppf topos. As noted in Appendix A, this requires working with $\mathbf{D}(\mathrm{QCoh})$ rather than the non-sensical $\mathbf{D}_{\mathrm{QCoh}}(\mathscr{M}$ od $)$. Throughout $\S 3.1$, $\mathscr{X} \rightarrow X$ is a fixed $D$-gerbe, where $D$ is a closed subgroup of the multiplicative group $\mathbf{G}_{m}$. We write $C$ for the dual of $D$; thus, $C$ is a quotient group of $\mathbb{Z}$.

\subsubsection{Twisted sheaves.}

Definition 3.1.1.1. An $\mathscr{X}$-twisted sheaf is a sheaf $\mathscr{F}$ of (left) $\mathscr{O}_{\mathscr{X}}$-modules such that the natural inertial action $\mathscr{F} \times D_{\mathscr{X}} \rightarrow \mathscr{F}$ (see Definition 2.2.1.6) equals the right action associated to the left module action $D_{\mathscr{X}} \times \mathscr{F} \rightarrow \mathscr{F}$.

Twisted vector bundles of rank $n$ naturally arise as 'universal reductions of structure group' for $\mathrm{PGL}_{n}$-bundles; the gerbe classifying the twisting is precisely the coboundary of the $\mathrm{PGL}_{n}$-bundle in $\mathrm{H}^{2}\left(\mathbf{G}_{m}\right)$ arising from the standard presentation $1 \rightarrow \mathbf{G}_{m} \rightarrow \mathrm{GL}_{n} \rightarrow \mathrm{PGL}_{n} \rightarrow 1$. A similar $\boldsymbol{\mu}_{n}$-gerbe 


\section{LIEBLICH}

arises when one studies reduction of structure group to $\mathrm{SL}_{n}$. It is a helpful exercise to write these gerbes down explicitly (as solutions to moduli problems). This may also be found in [Gir71].

Remark 3.1.1.2. There are two other ways of describing twisted $\mathscr{O}$-modules which have arisen in the literature. Both may be found in Căldăraru's thesis [Cal00] (available electronically). In the case that a faithful (i.e. nowhere zero) locally free twisted sheaf $\mathscr{V}$ exists, one can apply the functor $\mathscr{H} o m(\mathscr{V}, \cdot)$ and conclude (via a fibered form of Morita equivalence described in [Lie04]) that the abelian category of twisted $\mathscr{O}$-modules is equivalent to the abelian category of right $\mathscr{A}$-modules, where $\mathscr{A}=\mathscr{H} o m(\mathscr{V}, \mathscr{V})$.

Alternatively, one can give a cocyclic description of twisted sheaves (used by Căldăraru in his thesis [Cal00], Yoshioka in his recent work [Yos03], and others of a mathematico-physical bent) as follows: choose a hypercovering $U_{\bullet} \rightarrow X$ with a 2-cocycle $a \in \boldsymbol{\mu}_{n}\left(U_{2}\right)$ representing the cohomology class $[\mathscr{X}] \in \mathrm{H}^{2}\left(X, \boldsymbol{\mu}_{n}\right)$. (One can laboriously check [Lie04] that indeed choosing such a setup is tantamount to choosing $\mathscr{X}$.) A twisted sheaf is then a pair $(\mathscr{F}, \varphi)$, with $\mathscr{F}$ a sheaf of $\mathscr{O}_{U_{0}}$-modules and $\varphi: \operatorname{pr}_{1}^{*} \mathscr{F} \rightarrow \operatorname{pr}_{0}^{*} \mathscr{F}$ is an isomorphism on $U_{1}$ whose coboundary $\delta \varphi=a \in \operatorname{Aut}_{00}^{*} \mathscr{F}$ on $U_{2}$. In the cases where this definition has been applied to date, $X$ is usually quasi-projective over an affine, so it suffices to take a Čech hypercovering (by a theorem of Artin [Art71]) in the étale topology. (In fact, authors using this definition usually work over the complex numbers and use the classical topology.)

3.1.1.3 Since a $D$-gerbe is an Artin stack, there is a good theory of quasi-coherent and coherent twisted sheaves. Not surprisingly, this theory is influenced by the representation theory of $D$.

Given $\chi \in C$, for any quasi-coherent sheaf $\mathscr{F}$ on $\mathscr{X}$ there is a $\chi$-eigensheaf $\mathscr{F} \chi \subset \mathscr{F}$ corresponding to sections where the action of the inertia stack $A_{\mathscr{X}}$ is via the character $\chi: A \rightarrow \mathbf{G}_{m}$ and the right $\mathbf{G}_{m}$-action associated to the left $\mathscr{O}$-module structure of $\mathscr{F}$.

Proposition 3.1.1.4. Suppose that $\mathscr{F}$ is a quasi-coherent sheaf on $\mathscr{X}$. The natural maps induce an isomorphism

$$
\bigoplus_{\chi \in C(X)} \mathscr{F}_{\chi} \stackrel{\sim}{\rightarrow} \mathscr{F} .
$$

The eigensheaves $\mathscr{F}_{\chi}$ are quasi-coherent.

Proof. Using the specified identification $D_{\mathscr{X}} \cong \mathscr{I}(\mathscr{X})$ (which is part of the data describing a gerbe), the natural action of $\mathscr{I}(\mathscr{X})$ on $\mathscr{F}$ makes $\mathscr{F}$ a sheaf of quasi-coherent comodules for the Hopf algebra structure on $\mathscr{O}_{D_{\mathscr{X}}}$. Thus, the proposition immediately follows from the basic representation theory of diagonalizable group schemes [Wat79].

Let $\pi: Y \rightarrow X$ be a quasi-compact $X$-space and $\mathscr{Y}:=Y \times_{X} \mathscr{X} \rightarrow Y$ the pullback $D$-gerbe. We also denote the morphism $\mathscr{Y} \rightarrow \mathscr{X}$ by $\pi$.

LEMma 3.1.1.5. If $\mathscr{G}$ is a quasi-coherent sheaf on $\mathscr{Y}$, then the natural map $\pi_{*}\left(\mathscr{G}_{\chi}\right) \rightarrow \pi_{*} \mathscr{G}$ identifies $\pi_{*}\left(\mathscr{G}_{\chi}\right)$ with $\left(\pi_{*} \mathscr{G}\right)_{\chi}$.

Proof. As in the proof of Proposition 3.1.1.4, we can view $\mathscr{G}$ as an $\mathscr{O}_{D_{\mathscr{Y}}}$-comodule. In fact, the projection formula shows that the comodule structure on $\mathscr{G}$ is the adjoint of the induced $\mathscr{O}_{D \mathscr{X}^{-}}$ comodule structure on $\pi_{*} \mathscr{G}$. The result follows.

Definition 3.1.1.6. An $m$-fold $\mathscr{X}$-twisted sheaf is a sheaf $\mathscr{F}$ on $\mathscr{X}$ such that $\mathscr{F}=\mathscr{F}_{\chi_{m}}$, where $\chi_{m}: D \rightarrow \mathbf{G}_{m}$ is the $m$ th power of the natural character $D \rightarrow \mathbf{G}_{m}$.

Thus, $\mathscr{X}$-twisted sheaves are precisely the 1 -fold $\mathscr{X}$-twisted sheaves. Moreover, if $\mathscr{X}_{n} \rightarrow X$ represents the cohomology class $n[\mathscr{X}]$, then one can check (e.g. using the cocyclic formalism) 


\section{TWISTED SHEAVES AND THE PERIOD-INDEX PROBLEM}

that the category of $\mathscr{X}_{n}$-twisted sheaves is (non-canonically) equivalent to the category of $n$-fold $\mathscr{X}$-twisted sheaves. The reason to introduce $m$-fold twisted sheaves is for convenient bookkeeping, as the following lemma illustrates.

Lemma 3.1.1.7. If $\mathscr{F}$ is $m$-fold twisted and $\mathscr{G}$ is $n$-fold twisted, then:

(i) all cohomology sheaves of $\mathscr{F} \stackrel{\mathbf{L}}{\otimes} \mathscr{G}$ are $(n+m)$-fold twisted;

(ii) all cohomology sheaves of $\mathbf{R} \mathscr{H} o m(\mathscr{F}, \mathscr{G})$ are $(n-m)$-fold twisted.

If $\mathscr{X}$ is a $\boldsymbol{\mu}_{n}$-gerbe, then $n$-fold $\mathscr{X}$-twisted sheaves are naturally identified with 0 -fold $\mathscr{X}$-twisted sheaves, which are naturally identified via pushforward with sheaves on $X$.

Proof. This is practically a tautology.

Lemma 3.1.1.8. Given a $\mathbf{G}_{m}$-gerbe $\mathscr{Z} \rightarrow X$, there is an invertible $n$-fold $\mathscr{Z}$-twisted sheaf if and only if $n[\mathscr{Z}]=0 \in \mathrm{H}^{2}\left(X, \mathbf{G}_{m}\right)$. In particular, if there is a locally free $\mathscr{Z}$-twisted sheaf of rank $r>0$, then $r[\mathscr{Z}]=0 \in \mathrm{H}^{2}\left(X, \mathbf{G}_{m}\right)$.

Proof. The last statement follows by taking the determinant (Theorem A.3) and applying the first statement. To prove the first statement, we see from the fact that $\mathscr{X}_{n}$-twisted sheaves are identified with $n$-fold $\mathscr{X}$-twisted sheaves that it suffices to prove the statement assuming $n=1$. It follows from standard techniques that the groupoid of invertible sheaves on $\mathscr{X}$ is equivalent to the groupoid of 1 -morphisms $\mathscr{X} \rightarrow \mathbf{B G}_{m}$. (However, the reader should note that this is not the definition of $\mathbf{B G}_{m}$, which only involves classifying invertible sheaves on algebraic spaces - or perhaps only on affine schemes, depending upon one's ideological purity.) The condition that an invertible sheaf $\mathscr{L}$ on $\mathscr{X}$ be $\mathscr{X}$-twisted is easily seen to correspond to the condition that the corresponding morphism $\varphi_{\mathscr{L}}: \mathscr{X} \rightarrow \mathrm{BG}_{m, X}$ induces the identity on inertia stacks via the specified morphisms $\mathscr{I}(\mathscr{X}) \stackrel{\sim}{\rightarrow} \mathbf{G}_{m, \mathscr{X}} \rightarrow \varphi_{\mathscr{L}}^{*} \mathscr{I}\left(\mathrm{BG}_{m}\right)$. In other words, $\varphi \mathscr{L}$ is a morphism of $\mathbf{G}_{m^{-}}$ gerbes, hence an isomorphism, which means that the cohomology class of $\mathscr{X}$ is trivial. Conversely, if $\mathscr{X} \cong \mathbf{B G}_{m, X}$, then there is an $\mathscr{X}$-twisted invertible sheaf arising from the canonical one-dimensional representation of $\mathbf{G}_{m}$.

Proposition 3.1.1.9. If $X$ is Noetherian, then a quasi-coherent $\mathscr{X}$-twisted sheaf is the colimit of its coherent $\mathscr{X}$-twisted subsheaves.

Proof. This follows from the fact that $\mathscr{X}$ is Noetherian, combined with standard results about quasicoherent sheaves on Noetherian Artin stacks [LM00, $\S 15]$, along with the fact that any subsheaf of an $\mathscr{X}$-twisted sheaf is $\mathscr{X}$-twisted.

3.1.1.10 We end this section with several results comparing various categories of twisted sheaves.

Giving a global section of $\pi: \mathscr{X} \rightarrow X$ yields a 'trivialization' of the category of twisted sheaves.

Lemma 3.1.1.11. Given a section $\sigma: X \rightarrow \mathscr{X}$, the functor $\sigma^{*}$ defines an equivalence of categories between the category of $\mathscr{X}$-twisted sheaves and the category of $\mathscr{O}_{X}$-modules. This equivalence induces equivalences of the categories of quasi-coherent and coherent sheaves.

Proof. Given $\sigma$, there results an isomorphism $\widetilde{\sigma}: \mathscr{X} \stackrel{\sim}{\rightarrow} \mathrm{B} D$ of $D$-gerbes on $X$ which sends an object $\tau$ to $\operatorname{Isom}(\tau, \sigma)$. The natural character of $D$ gives an invertible $\mathrm{B} D$-twisted sheaf which pulls back to yield an invertible $\mathscr{X}$-twisted sheaf $\mathscr{L}$. The functor $\mathscr{W} \mapsto\left(\pi^{*} \mathscr{W}\right) \otimes \mathscr{L}$ gives an equivalence between the category of $\mathscr{O}_{X}$-modules (with the inverse given by $\mathscr{V} \mapsto \pi_{*}\left(\mathscr{V} \otimes \mathscr{L}^{\vee}\right)$.

The reader more comfortable with cocycles can also give a proof of this statement using cocyclic description of Remark 3.1.1.2. 


\section{LIEBLICH}

Using Lemma 3.1.1.11, we can prove that the category of twisted sheaves is invariant under a 'change of groups'. Suppose $\alpha \in \mathrm{H}^{2}\left(X, \boldsymbol{\mu}_{n}\right)$, and let $\iota_{*} \alpha$ be the image under the natural map $\mathrm{H}^{2}\left(X, \boldsymbol{\mu}_{n}\right) \rightarrow \mathrm{H}^{2}\left(X, \mathbf{G}_{m}\right)$ induced by the inclusion $\boldsymbol{\mu}_{n} \hookrightarrow \mathbf{G}_{m}$. There is a $\boldsymbol{\mu}_{n}$-gerbe $\mathscr{Z} \rightarrow X$ and a $\mathbf{G}_{m}$-gerbe $\mathscr{Y} \rightarrow X$ with a $\boldsymbol{\mu}_{n}$-linear map $\widetilde{\iota}: \mathscr{Z} \rightarrow \mathscr{Y}$ over $X$.

Lemma 3.1.1.12. The pullback functor associated to the morphism $\widetilde{\iota}$ yields an equivalence of the category of $\mathscr{Y}$-twisted sheaves with the category of $\mathscr{Z}$-twisted sheaves. This equivalence induces equivalences of categories of quasi-coherent and coherent sheaves.

Proof. Since $\mathscr{Z}$-twisted sheaves and $\mathscr{Y}$-twisted sheaves both form stacks on $X$, it suffices to prove the statement after covering $X$ so that both gerbes become trivial. Let $\mathscr{L}$ be an invertible $\mathscr{Y}$ twisted sheaf coming from a choice of section as in Lemma 3.1.1.11. Given a sheaf $\mathscr{W}$ on $X$, there is a natural isomorphism $\widetilde{\iota}^{*}\left(\pi_{\mathscr{Y}}^{*} \mathscr{W} \otimes \mathscr{L}\right) \stackrel{\sim}{\rightarrow} \pi_{\mathscr{L}}^{*} \mathscr{W} \otimes \widetilde{\iota}^{*} \mathscr{L}$, and this immediately implies the result.

One can also give a proof using the cocyclic description of twisted sheaves given in Remark 3.1.1.2: the image in $\mathbf{G}_{m}$ of the cocycle for $\boldsymbol{\mu}_{n}$ corresponding to $\mathscr{X}$ gives the cocycle corresponding to $\mathscr{Y}$. The cocyclic description of twisted sheaves now makes it clear that the categories are equivalent.

3.1.2 Twisted interpretations of $\mathrm{Br}=\mathrm{Br}^{\prime}$ and period-index. Using the technology of twisted sheaves, we can now clearly state both the equality of $\mathrm{Br}$ and $\mathrm{Br}^{\prime}$ and the period-index problem in purely geometric terms. We assume in this section that $X$ is integral and Noetherian and that $D=\boldsymbol{\mu}_{n}$.

Proposition 3.1.2.1. Let $\mathscr{X} \rightarrow X$ be a $\mathbf{G}_{m}$-gerbe and $m$ a positive integer:

(i) $[\mathscr{X}]$ lies in $\operatorname{Br}(X)$ if and only if there is a non-zero locally free $\mathscr{X}$-twisted sheaf;

(ii) if $X=\operatorname{Spec} K$ is a reduced point, then ind[ $\mathscr{X}]$ divides $m$ if and only if there is a coherent $\mathscr{X}$-twisted sheaf of rank $m$;

(iii) if $X$ is regular of dimension at most two, then ind $\left[\left.\mathscr{X}\right|_{\kappa(X)}\right]$ divides $m$ if and only if there is a locally free $\mathscr{X}$-twisted sheaf of rank $m$;

(iv) the class $[\mathscr{X}] \in \mathrm{H}^{2}\left(X, \boldsymbol{\mu}_{n}\right)$ lies in the image of the coboundary map $\mathrm{H}^{1}\left(X, \mathrm{PGL}_{n}\right) \rightarrow \mathrm{H}^{2}\left(X, \boldsymbol{\mu}_{n}\right)$ if and only if there is a locally free $\mathscr{X}$-twisted sheaf of rank $n$ and trivial determinant (Theorem A.3).

Sketch of proof. The relevant fact is the following: there is a locally free $\mathscr{X}$-twisted sheaf of $\operatorname{rank} r$ if and only if there is an Azumaya algebra of degree $r$ with class [ $\mathscr{X}]$. The equivalence arises by sending $\mathscr{V}$ to $\mathscr{E} n d(\mathscr{V})$. The inverse comes by associating to an Azumaya algebra $\mathscr{A}$ its gerbe of trivializations: a section over a scheme $Y \rightarrow X$ is a locally free sheaf $\mathscr{W}$ along with an isomorphism of algebras $\mathscr{E} n d(\mathscr{W}) \stackrel{\sim}{\rightarrow} \mathscr{A}$. It is easy to check that this is a $\mathbf{G}_{m}$-gerbe with class $[\mathscr{A}] \in \operatorname{Br}(X) \subset \mathrm{H}^{2}\left(X, \mathbf{G}_{m}\right)$, and that the sheaves $\mathscr{W}$ patch together to yield a locally free twisted sheaf of rank $n$. This is essentially carried out in [Gir71, §V.4]. The third statement follows from the second, combined with Proposition 3.1.3.9.

To prove the fourth statement, suppose that $\mathscr{V}$ is a locally free $\mathscr{X}$-twisted sheaf of rank $n$, so that $\mathscr{E} n d(\mathscr{V})$ is the pullback of an Azumaya algebra $\mathscr{A}$ of degree $n$ on $X$. It is a simple calculation to show that the class of $\mathscr{A}$ differs from $\mathscr{X}$ by the image of det $\mathscr{V}$ in $\mathrm{H}^{2}\left(X, \boldsymbol{\mu}_{n}\right)$ via the 'first Chern class' map. Thus, the difference vanishes if and only if $\operatorname{det} \mathscr{V}$ is an $n$th power, in which case we can twist $\mathscr{V}$ to produce a locally free $\mathscr{X}$-twisted sheaf of rank $n$ with trivial determinant.

Thus, these become problems about the existence of points in various moduli spaces: locally free (or simply coherent) twisted sheaves (of a given rank). The period-index problem in particular will prove amenable to this kind of analysis. The question of $\mathrm{Br}$ and $\mathrm{Br}^{\prime}$ is more profitably approached in terms of the $K$-theory of $\mathscr{X}$, as we describe in $\S 3.1 .4$. 


\section{TWISTED SHEAVES AND THE PERIOD-INDEX PROBLEM}

3.1.3 Elementary applications. In this section, we assume that $X$ is Noetherian and that $D=\mathbf{G}_{m}$.

Here we investigate the applications of the theory we have developed so far to the study of the Brauer group. While seemingly vacuous, the theory of twisted sheaves yields many of the basic results on the Brauer group without requiring recourse to étale cohomology. (The skeptical reader should note that some of the fundamental properties of gerbes and twisted sheaves do rely on the relationship with cohomology, but this is where the similarity ends. The point is that we use sheaftheoretic methods in place of explicit computations of cohomological dimensions and the use of cohomological purity.) The essential result for all of these proofs, it turns out, is the deceptively simple-looking Proposition 3.1.1.9.

Lemma 3.1.3.1. Let $\eta \subset X$ be the scheme of generic points. Any coherent $\mathscr{X}_{\eta}$-twisted sheaf extends to a coherent $\mathscr{X}$-twisted sheaf.

Proof. Let $\mathscr{F}$ be a coherent $\mathscr{X}_{\eta}$-twisted sheaf. The inclusion $\nu: \mathscr{X}_{\eta} \hookrightarrow \mathscr{X}$ is quasi-compact, hence $\nu_{*} \mathscr{F}$ is a quasi-coherent $\mathscr{X}$-twisted sheaf. The result follows from Proposition 3.1.1.9.

Lemma 3.1.3.2. There exists a non-zero coherent $\mathscr{X}$-twisted sheaf with support $\mathscr{X}$.

Proof. Over the reduced structure on the generic scheme of $X$, we have a $\mathbf{G}_{m}$-gerbe over the spectrum of a finite product of fields. Thus, if we can produce a non-zero coherent twisted sheaf when $X$ is the spectrum of a field, we can push it forward to get such an object on the generic scheme of $X$ and then apply Lemma 3.1.3.1. When $X$ is Spec $K$, any étale covering is finite over $X$. Thus, there is a finite free morphism $Y \rightarrow X$ such that the gerbe $\mathscr{Y}:=Y \times_{X} \mathscr{X}$ has a section. Once there is a section, we can apply Lemma 3.1.1.11. Thus, there is a non-zero (in fact, locally free) twisted sheaf on $\mathscr{Y}$. Pushing forward along the morphism $\mathscr{Y} \rightarrow \mathscr{X}$ yields a non-zero locally free $\mathscr{X}$-twisted sheaf by Lemma 3.1.1.5. (For a generalization of this argument, see Lemma 3.1.3.5.)

Proposition 3.1.3.3. If $X$ is regular and integral with generic scheme $\eta$, then the restriction map

$$
\mathrm{H}^{2}\left(X, \mathbf{G}_{m}\right) \rightarrow \mathrm{H}^{2}\left(\eta, \mathbf{G}_{m}\right)
$$

is an injection.

Proof. Suppose that $\mathscr{X} \times_{X} \eta$ represents the trivial cohomology class. This means that there is an invertible $\mathscr{X}_{\eta}$-twisted sheaf $L_{\eta}$. By Proposition 3.1.1.9, $L_{\eta}$ has a coherent extension $L$ on all of $\mathscr{X}$. In fact, there is a reflexive such extension. On the other hand, this extension has rank one by construction. However, $X$, and therefore $\mathscr{X}$, is regular. As any reflexive module of rank one over a regular local ring (of arbitrary dimension) is free [Bou89, $\S$ VII.4], we conclude that $L$ is an invertible twisted sheaf, whence $[\mathscr{X}]=0 \in \mathrm{H}^{2}\left(X, \mathbf{G}_{m}\right)$.

Corollary 3.1.3.4. If $X$ is regular and quasi-compact, then $\mathrm{H}^{2}\left(X, \mathbf{G}_{m}\right)$ is torsion.

Proof. This follows immediately from Proposition 3.1.3.3 and the fact that the Galois cohomology of a field with abelian coefficients is torsion.

Before proceeding, we recall a couple of lemmas which are also useful in $§ 3.1 .4$.

Lemma 3.1.3.5. If $Y \rightarrow X$ is a finite locally free covering and $\alpha \in \mathrm{H}^{2}\left(X, \mathbf{G}_{m}\right)$, then there is a nowhere zero locally free twisted sheaf on $Y$ if and only if there is such a twisted sheaf on $X$. If $X$ is quasi-compact, the same holds for locally free twisted sheaves of finite constant non-zero rank.

Proof. Fixing a gerbe $\mathscr{X}$ representing $\alpha$, we see that $\mathscr{Y}:=\mathscr{X} \times_{X} Y$ represents the pullback of $\alpha$ to $Y$. Furthermore, $\pi: \mathscr{Y} \rightarrow \mathscr{X}$ is a finite locally free morphism. Thus, given any locally free twisted sheaf $F$ on $\mathscr{Y}, \pi_{*} F$ will be a locally free twisted sheaf on $\mathscr{X}$. It is clear that the rank of 


\section{LIEBLICH}

such a sheaf will be constant on a connected component. If $X$ is quasi-compact, then there are only finitely many connected components, whence one can arrange for the rank to be constant by taking appropriate direct sums on each component.

Lemma 3.1.3.6 (Gabber). Given a local ring $A$ and a local-étale $A$-algebra $B$, there exists a finite free $A$-algebra $C$ such that for all maximal ideals $\mathfrak{m} \subset C$, there is a map $B \rightarrow C_{\mathfrak{m}}$ of $A$-algebras.

Sketch of proof, following Gabber. By the local structure theory for étale morphisms [Ray70], there is a monic polynomial $f(x)=x^{n}+\sum_{i=0}^{n-1} a_{i} x^{n-i} \in A[x]$ and a maximal ideal $\mathfrak{n} \subset A[x]$ such that $f^{\prime} \notin \mathfrak{n}$ and $B \cong(A[x] / f(x))_{\mathfrak{n}}$. Let $C$ be $A\left[T_{1}, \ldots, T_{n}\right] /\left(\left\{\sigma_{i}\left(T_{1}, \ldots, T_{n}\right)-(-1)^{i} a_{i} ; i=1, \ldots, n\right\}\right)$, where $\sigma_{i}$ is the $i$ th symmetric polynomial. One can check that $C$ is a finite free $A$-module. By construction, we have that $f(x)=\prod\left(x-T_{i}\right)$ in $C[x]$, so that there are factorizations $\varphi_{i}: A[x] /(f(x)) \rightarrow C$ over $A$ given by sending $x$ to $T_{i}$. It is possible to see that $C$ is a finite free $A[x] /(f(x))$-module of rank $(n-1)$ ! via $\varphi_{1}$, from which it follows that there is some maximal ideal $\mathfrak{q} \subset C$ lying over $\mathfrak{n} \subset A[x] /(f(x))$, yielding a homomorphism $B \rightarrow C_{\mathfrak{q}}$. There is an obvious action of the symmetric group $\Sigma_{n}$ on $C$ over $A$, which is transitive on the closed fiber. Thus, composing the $A$-morphism $B \rightarrow C_{\mathfrak{q}}$ with the automorphisms arising from the action of $\Sigma_{n}$, we see that there is in fact an $A$-map $B \rightarrow C_{\mathfrak{m}}$ for every maximal ideal $\mathfrak{m} \subset C$, as desired.

Proposition 3.1.3.7. If $X$ is semilocal of dimension at most one, then there exists a locally free $\mathscr{X}$-twisted sheaf of positive rank.

Proof. When the cohomology class of $\mathscr{X}$ is assumed to be torsion, this is subsumed by Theorem 3.1.4.1; thus, we only give a sketch here.

We may assume that $X$ is connected. It suffices to prove the statement assuming that $X$ is reduced. Indeed, the infinitesimal deformations of a locally free $\mathscr{X}_{\text {red }}$-twisted sheaf are easily seen to be unobstructed (using the fact that coherent cohomology vanishes on an affine scheme), so that

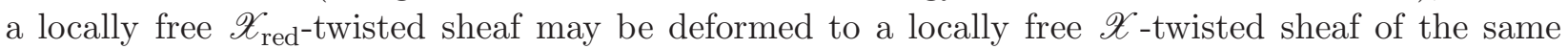
rank.

If $X$ has dimension zero, then it is a disjoint union of reduced closed points. Using the fact that the Brauer group and cohomological Brauer group coincide over fields [Ser95, § X.5]) and elementary (e.g. Morita-theoretic) considerations, we see that there are locally free $\mathscr{X}$-twisted sheaves and that moreover there is at most one isomorphism class of $\mathscr{X}$-twisted sheaves with a given rank (at each point). (Briefly, the point is that by Morita theory one can see that two such differ by tensoring with an invertible sheaf, which is trivial over a semilocal ring.)

Now suppose that $X$ is local. Applying Lemmas 3.1.3.5 and 3.1.3.6, we may replace $X$ by a finite semilocal extension $Y \rightarrow X$ such that $\mathscr{Y}:=\mathscr{X} \times_{X} Y$ is trivial over every localization of $Y$. Choosing trivializations of the gerbe at each closed point of $Y$, it is immediate that there exists an integer $N$ such that for any closed point $y \in Y$, there is a locally free twisted sheaf on $\operatorname{Spec} \mathscr{O}_{y, Y}$ of rank $N$. By the previous paragraph, these are all isomorphic on the generic scheme of $Y$ (as $y$ varies). It follows (since $Y$ is of dimension one at each closed point) that we can glue the local twisted sheaves to produce a locally free $\mathscr{Y}$-twisted sheaf.

The general case proceeds as in the previous paragraph: the complement of the set of closed points of $X$ is open (since the dimension is at most one at any point), so local models can be glued together to yield a locally free $\mathscr{X}$-twisted sheaf.

Corollary 3.1.3.8. If $X$ is a semilocal one-dimensional Noetherian scheme, $\mathrm{H}^{2}\left(X, \mathbf{G}_{m}\right)$ is torsion and equals $\operatorname{Br}(X)$.

Proof. This is an application of Proposition 3.1.2.1, Lemma 3.1.1.8, and Proposition 3.1.3.7. 


\section{TWISTED SHEAVES AND THE PERIOD-INDEX PROBLEM}

Proposition 3.1.3.9. If $X$ is Noetherian there is a coherent twisted sheaf which is locally free at every point of codimension one. If $X$ is regular, there is a coherent twisted sheaf which is locally free at every point of codimension two.

Proof. Using the first half of the proof of Proposition 3.1.3.7, it is easy to find some open $V \subset X$ over which there is a locally free twisted sheaf $F$. Suppose that $p \in X \backslash V$ has codimension one in $X$. Let $i:$ Spec $\mathscr{O}_{p, X} \hookrightarrow X$ and $j: V \hookrightarrow X$. By Proposition 3.1.3.7, we may assume (by taking a direct sum of $F$ with itself if necessary) that there exists a locally free twisted sheaf $G$ on $\operatorname{Spec} \mathscr{O}_{p, X}$ such that $G_{\eta}=F_{\eta}$ (identifying the generic schemes of Spec $\mathscr{O}_{p, X}$ and $V$ with $\eta$ using $i$ and $j$, respectively). Consider $Q:=i_{*} G \cap j_{*} F \subset F_{\eta}$. This is a quasi-coherent twisted sheaf on $X$ which equals $F$ when pulled back by $j$ and $G$ when pulled back by $i$. Applying Proposition 3.1.1.9 and the fact that localization commutes with colimits, we see that there is a coherent subsheaf $P \subset Q$ such that $\left.P\right|_{V} \cong F$ and $\left.P\right|_{\text {Spec } \mathscr{O}_{p, X}} \cong G$. The locus where $P$ is locally free is thus an open set containing $V \cup\{p\}$. By Noetherian induction, the first statement is proven. (More concretely, there can only be finitely many codimension one points $p$ not in $V$, as they must be generic points of irreducible components of $X \backslash V$.) The second statement follows from the fact that any reflexive module over a regular local ring of dimension at most two is free. Thus, the reflexive hull of any coherent twisted sheaf will be locally free in codimension two.

Corollary 3.1.3.10. If $X$ is regular of dimension at most two everywhere, then the inclusion $\operatorname{Br}(X) \hookrightarrow \mathrm{H}^{2}\left(X, \mathbf{G}_{m}\right)$ is an isomorphism.

Proof. This follows from Proposition 3.1.3.9 and Lemma 3.1.1.8.

3.1.4 Gabber's theorems. We wish to show how twisted sheaves may be used to give an especially streamlined proof of Gabber's theorem that $\mathrm{Br}=\mathrm{Br}^{\prime}$ for affine schemes. (In fact, the proof given here also works for separated unions of two affines, just as in Gabber's original result.) Our argument is a simplification of the argument of Hoobler [Hoo82] which is itself a simplification of Gabber's proof. By using twisted sheaves rather than Azumaya algebras, the introduction of $K$ theory into the proof becomes more transparent, as one can 'think in modules' from the beginning. Our approach notably also avoids the comparison of the 'Mayer-Vietoris sequence' in non-abelian flat cohomology with that in ordinary abelian flat cohomology, by absorbing all of the cohomology (abelian and otherwise) into the underlying gerbe $\mathscr{X}$. The outline of our proof comes from Hoobler's paper [Hoo82].

The main theorem of this section is the following.

Theorem 3.1.4.1. Let $X$ be an affine scheme and $\mathscr{X}$ an fppf $\boldsymbol{\mu}_{n}$-gerbe on $X$. There exists an $\mathscr{X}$-twisted locally free sheaf of constant finite non-zero rank.

Corollary 3.1.4.2. If $X$ is an affine scheme, then the natural injection $\operatorname{Br}(X) \hookrightarrow \operatorname{Br}^{\prime}(X)$ is an isomorphism.

Proof. Any torsion class $\alpha \in \mathrm{H}^{2}\left(X, \mathbf{G}_{m}\right)$ (taken in the flat topology or étale topology) has a lift to a flat cohomology class in $\mathrm{H}^{2}\left(X, \boldsymbol{\mu}_{n}\right)$. The theorem gives a twisted vector bundle on a gerbe in any such class. Taking its endomorphism algebra yields an Azumaya algebra with class $\alpha$.

For the rest of this section, we assume that $X$ is an affine scheme and $\mathscr{X} \rightarrow X$ is a $\boldsymbol{\mu}_{n^{-}}$ gerbe.

The proof of Theorem 3.1.4.1 follows from several basic $K$-theoretic lemmas. Let $R$ be a commutative unital ring and let $K(R)$ denote the ring structure on the Grothendieck group of finite projective $R$-modules of constant rank. We recall some basic facts from algebraic $K$-theory. 


\section{LIEBLICH}

Lemma 3.1.4.3. There is a natural map $\rho: K(R) \rightarrow \mathbb{Z}$ determined by the rank function on modules.

(i) The kernel of $\rho$ is a nil-ideal.

(ii) Given projective modules $P$ and $Q$, if $[P]=[Q]$ in $K(R)$, then there is $N>0$ such that $P^{\oplus N} \cong Q^{\oplus N}$

(iii) Given a class $\alpha \in K(R)$ of positive rank, some multiple n $\alpha$ has the form $[P]$ for a projective module $P$.

(iv) If $P$ is a projective $R$-module such that $P^{\otimes n}$ is free, then there is some $N>0$ such that $P^{\oplus N}$ is free.

Sketch of proof. Since the $K$-group classifies finitely generated projective modules, we immediately have that it commutes with colimits in $R$. Thus, to prove any statement involving only finitely many elements it suffices to prove it assuming that $R$ is finitely generated over $\mathbb{Z}$. In this case, $R$ has some Krull dimension $d$.

A beautiful proof of the first statement is due to Gabber and may also be found in his thesis [Gab81]. It is easy to see that $\operatorname{ker} \rho$ is generated by elements of the form $[P]-\left[R^{\oplus \operatorname{rk} P}\right]$, and thus that it suffices to prove the statement for such elements. Now we can consider complexes $0 \rightarrow R^{\oplus \operatorname{rk} P} \rightarrow P \rightarrow 0$, given by a morphism $\varphi_{i}$, and it is easy to see that there are precisely $d$ such complexes such that for any point $p \in \operatorname{Spec} R$, one of the complexes is exact at $p$ (i.e. one of the $\varphi_{i}$ gives a trivialization of $P$ in a neighborhood of $p$ ). However, then the tensor product of the complexes $\varphi_{1} \otimes \cdots \otimes \varphi_{d}$ is exact, whence it vanishes in $K$-theory. It follows that $\left([P]-\left[R^{\oplus \operatorname{rk} P}\right]\right)^{d}=0$ in $K(R)$.

The second statement is quite a bit more difficult, and relies on the Bass cancellation lemma. (In the commutative case, it ultimately derives from a simpler statement due to Serre, which states that any projective module of rank at least $d+1$ has a non-zero free summand.) This is beyond the scope of this paper; we refer the reader to [Bas68, $\S 4$ of Chapter IX] for the details. (The reader will also find a slightly different account of the rest of the $K$-theory we use here, including the other statements contained in this lemma.)

The third statement may be proven as follows. First, we may assume $R$ has finite Krull dimension $d$. Scaling $\alpha$, we may assume that $\operatorname{rk} n \alpha \geqslant d$. We can certainly write $n \alpha=[P]-[Q]$ for some projective modules $P$ and $Q$. Choosing a representation $R^{N}=Q \oplus Q^{\prime}$, we find that $n \alpha+N=\left[P \oplus Q^{\prime}\right]$. However,

$$
\operatorname{rk}\left(P \oplus Q^{\prime}\right)=\operatorname{rk}(n \alpha+N) \geqslant d+N
$$

so we can find a free summand of $P \oplus Q^{\prime}$ of rank $N$ by Serre's theorem. The complementary summand yields a projective module representing $n \alpha$, as desired.

To prove the final statement, let $r$ be the rank of $P$, and write $[P]=r+\eta \in K(R)$; we know from the first statement that $\eta$ is nilpotent. By hypothesis, $(r+\eta)^{n} \in \mathbb{Z} \subset K(R)$, and it follows from the nilpotence of $\eta$ that $n \eta=0$, so that $\left[P^{\oplus n}\right]=\left[R^{n r}\right]$. Applying the second part, we conclude that there is $N$ such that $P^{\oplus N}$ is free, as required.

Corollary 3.1.4.4. Given a faithfully projective $R$-module $P$ and a positive integer $n$, there exist non-zero free modules $F_{0}$ and $F_{1}$ and a faithfully projective $R$-module $\bar{P}$ such that $P \otimes \bar{P}^{\otimes n} \otimes F_{0} \cong F_{1}$.

Proof. In $K$-theory, the desired equality reads $a[P][\bar{P}]^{n}=b$ for some positive integers $a$ and $b$. In fact, applying Lemma 3.1.4.3(iii), we see that it is enough to solve the equation for $[\bar{P}]$ in $K(R)$. Moreover, since we are allowed to scale by $a$ and $b$, it is clearly enough to solve the equation in $K(R) \otimes \mathbf{Q}$. Writing $[P]=a+\beta$ with $\beta \in \operatorname{ker} \rho$, we see that since $\beta$ is nilpotent we can invert $(1 / a)[P]$ and extract an $n$th root using formal power series with rational coefficients, thus yielding a solution in $K(R) \otimes \mathbf{Q}$. 


\section{TWISTED SHEAVES AND THE PERIOD-INDEX PROBLEM}

Combining Lemmas 3.1.3.6 and 3.1.3.5, we see that to prove Theorem 3.1.4.1 it is enough to prove the following. We use the phrase 'locally free' to mean 'locally free of constant non-zero rank' in what follows.

Proposition 3.1.4.5. Suppose that there is everywhere Zariski-locally on $X$ a locally free $\mathscr{X}$-twisted sheaf. Then there is a global locally free $\mathscr{X}$-twisted sheaf.

Proof. To simplify the notation, throughout this proof we use the phrase 'twisted sheaf on $U$ ' to mean ' $\mathscr{X} \times_{X} U$-twisted sheaf' whenever $U \subset X$ is a subscheme. It is enough to show that

$$
J=\left\{f \in A \mid \text { there is a locally free twisted sheaf on } \operatorname{Spec} A_{f}\right\}
$$

is an ideal. The Zariski-local existence hypothesis shows that $J$ cannot be contained in any maximal ideal, hence if it is an ideal $J=A$. (This is Quillen induction.) It is clear that $J$ is closed under multiplication by elements of $A$. To check that $J$ is closed under addition, we may reduce to the case where $X=U \cup V$ with $U, V$, and $U \cap V$ all affine, and there are locally free twisted sheaves on $U$ and $V$. (Indeed, suppose $f, g \in J$; we wish to show that $f+g \in J$. We may replace $X$ by Spec $A_{f+g}$ and thus assume that $f+g=1$, in which case Spec $A_{f}$ and Spec $A_{g}$ form an open cover of $X$. Moreover, $\operatorname{Spec} A_{f} \cap \operatorname{Spec} A_{g}=\operatorname{Spec} A_{f g}$ is affine.)

Let $\mathscr{P}$ be a locally free twisted sheaf on $U$ and $\mathscr{Q}$ a locally free twisted sheaf on $V$; we may assume that rk $\mathscr{P}=$ rk $\mathscr{Q}$. Since $\mathscr{X}$ is a $\boldsymbol{\mu}_{n}$-gerbe, we see that $\mathscr{P} \otimes n$ is naturally identified with a locally free untwisted sheaf (see Lemma 3.1.1.7), and similarly for $\mathscr{Q}$. By Corollary 3.1.4.4, we see that there are non-zero locally free sheaves $\bar{P}$ on $U, \bar{Q}$ on $V$ and non-zero finite free modules $F_{0}, F_{1}$ on $U, G_{0}, G_{1}$ on $V$ such that

$$
\mathscr{P}^{\otimes n} \otimes \bar{P}^{\otimes n} \otimes F_{0} \cong F_{1}
$$

and

$$
\mathscr{Q}^{\otimes n} \otimes \bar{Q}^{\otimes n} \otimes G_{0} \cong G_{1} .
$$

Thus, replacing $\mathscr{P}$ by $\mathscr{P} \otimes \bar{P} \otimes F_{0}$ and $\mathscr{Q}$ by $\mathscr{Q} \otimes \bar{Q} \otimes G_{0}$, we may assume that $\mathscr{P} \otimes n$ and $\mathscr{Q}^{\otimes n}$ are free modules on $U$ and $V$, respectively, of the same rank. Now consider the situation on $U \cap V$. Letting $P=\mathscr{Q}^{\vee} \otimes \mathscr{Q}$ and $Q=\mathscr{P} \otimes \mathscr{Q}^{\vee}$, we have an isomorphism of locally free twisted sheaves $\mathscr{P} \otimes P \cong \mathscr{Q} \otimes Q$. Furthermore, taking $n$th tensor powers, we see that $P^{\otimes n}$ and $Q^{\otimes n}$ are both free (of the same rank). Applying Lemma 3.1.4.3(iv), we see that there is some $N>0$ such that $P^{\oplus N}$ and $Q^{\oplus N}$ are free. From the isomorphism $\mathscr{P} \otimes P \cong \mathscr{Q} \otimes Q$ we deduce that $\mathscr{P} \otimes P^{\oplus N} \cong \mathscr{Q} \otimes Q^{\oplus N}$, from which it follows that $\mathscr{P}^{M} \cong \mathscr{Q}^{M}$ for some integer $M>0$. Thus, $\mathscr{P}^{M}$ and $\mathscr{Q}^{M}$ are locally free twisted sheaves on $U$ and $V$ which glue on $U \cap V$, as desired.

Starting with the affine case (Corollary 3.1.4.2), it is possible to use twisted sheaves to prove the following.

Proposition 3.1.4.6. If $Y$ is a quasi-compact separated scheme admitting an ample invertible sheaf, then the natural injection $\operatorname{Br}(Y) \hookrightarrow \operatorname{Br}^{\prime}(Y)$ is an isomorphism.

The reader is referred to [Dej03] for the proof. The idea is to start with a supply of twisted sheaves which are locally free at selected points (using Proposition 3.1.4.5), and to increase the locus over which there exists a locally free twisted sheaf by looking at kernels of general maps between the local models. The ample invertible sheaf enables one to make a Bertini argument when studying a general such map between twisted sheaves (tensored with powers of the ample invertible sheaf).

\subsection{Moduli: a summary}

In this section we summarize the important aspects of the theory of moduli of twisted sheaves, a subject which is treated in full detail (with proofs) in [Lie07b]. In $\S 4$, we use the structure theory 


\section{LIEBLICH}

of these moduli spaces to prove results about the period-index problem for curves and surfaces over finite, algebraically closed, and local fields.

3.2.1 The moduli problem. Let $X \rightarrow S$ be a smooth projective morphism (with a chosen relatively ample $\mathscr{O}(1)$ ) with geometrically connected fibers, $n$ a positive integer which is invertible on $S$, and $\mathscr{X} \rightarrow X$ a $\boldsymbol{\mu}_{n}$-gerbe. Using standard methods, one can prove the following (see [Lie07b]).

Proposition 3.2.1.1. The stack $\mathbf{T w}_{\mathscr{X} / S}$ of $S$-flat torsion free $\mathscr{X}$-twisted sheaves is an Artin stack locally of finite presentation over $S$.

Given $T \rightarrow S$, the sections of $\mathbf{T w}_{\mathscr{X} / S}$ over $T$ are $T$-flat quasicoherent sheaves of finite presentation $\mathscr{F}$ on $\mathscr{X} \times{ }_{S} T$ which are $\mathscr{X} \times{ }_{S} T$-twisted and such that for every geometric point $t \rightarrow T$, the fiber $\mathscr{F}_{t}$ on $\mathscr{X}_{t}$ is torsion free. (The reader is referred to [Lie07b] for basic results on associated points and torsion subsheaves on Artin stacks. Nothing surprising happens.)

As in the case of ordinary sheaves on a projective morphism, the stack of all twisted sheaves is a nightmarish object. For this and other reasons, it is worthwhile to distinguish an open substack of semistable objects (and a further open substack of stable objects). In the cases at hand (namely for curves and surfaces), it will turn out that this stack is in fact a GIT quotient stack; however, the definition can be made in arbitrary dimension, and while the stack has all of the properties one expects of a GIT quotient stack, it is not clear whether it is in fact such a quotient stack.

To define the stability condition, we assume for the sake of simplicity that $S=$ Spec $k$ is the spectrum of a field. In this case, we can make use of the degree function $\operatorname{deg} A_{0}(\mathscr{X}) \rightarrow \mathbf{Q}$, which can be defined as follows, following [Vis89]. There is a rational Chow theory $A_{*}(\mathscr{X})$ for which there is a natural theory of Chern classes. Via proper pushforward, there is an isomorphism $A_{0}(\mathscr{X}) \rightarrow$ $A_{0}(X) \otimes \mathbf{Q}$.

Definition 3.2.1.2. The degree map deg $: A_{0}(\mathscr{X}) \rightarrow \mathbf{Q}$ is the composition $A_{0}(\mathscr{X}) \rightarrow A_{0}(X) \otimes$ $\mathbf{Q} \rightarrow \mathbf{Q}$, where the latter map is the degree in the usual Chow theory of $X$.

In the following, we write $\operatorname{Td} \mathscr{X}$ for the Todd class of the tangent sheaf of $\mathscr{X}$.

Definition 3.2.1.3. Given a coherent sheaf $\mathscr{F}$ on $\mathscr{X}$, the geometric Hilbert polynomial of $\mathscr{F}$, denoted $P_{\mathscr{F}}^{g}$, is the polynomial whose value at $m$ is $n \operatorname{deg}(\operatorname{ch}(\mathscr{F}(m)) \cdot \operatorname{Td} \mathscr{X})$.

It is a standard exercise to show that this in fact defines a (unique) polynomial over Q. Furthermore, one can see that for torsion free $\mathscr{F}$, the polynomial has degree equal to the dimension of $X$, with leading coefficient equal to $\mathrm{rk} \mathscr{F} \operatorname{deg}_{X} \mathscr{O}(1) /(\operatorname{dim} X)$ !.

Remark 3.2.1.4. When the base $S$ is arbitrary, one can check that in fact the geometric Hilbert polynomial of an $S$-flat $\mathscr{X}$-twisted sheaf is constant in geometric fibers. The reader is referred to [Lie07b, Remark 2.2.7.18] for the details.

With this additive function $K(\mathscr{X}) \rightarrow \mathbf{Q}[x]$ in hand, we define (semi)stability in the usual way. Recall that there is an ordering on $\mathbf{Q}[x]$ given by the lexicographical ordering of coefficients. In this ordering, $f \leqslant g$ if and only if $f(a) \leqslant g(a)$ for all sufficiently large (positive) integers $a$.

Definition 3.2.1.5. With the above notation, a coherent sheaf $\mathscr{F}$ on $\mathscr{X}$ is semistable if for all coherent proper subsheaves $\mathscr{G} \subset \mathscr{F}$, one has $(\mathrm{rk} \mathscr{F}) P_{\mathscr{G}}^{g} \leqslant(\operatorname{rk} \mathscr{G}) P_{\mathscr{F}}^{g}$. The sheaf $\mathscr{F}$ is stable if one has strict inequality for all coherent proper subsheaves $\mathscr{G} \subset \mathscr{F}$.

Remark 3.2.1.6. When the base $S$ is arbitrary, one can check that given an $S$-flat family $\mathscr{F}$ of coherent $\mathscr{X}$-twisted sheaves, there is an open subset of $S$ parametrizing semistable fibers $\mathscr{F}_{s}$ and a smaller open subset parametrizing geometrically stable fibers $\mathscr{F}_{s}$. The reader is referred to [Lie07b, Corollary 2.3.2.11]. 


\section{TWISTED SHEAVES AND THE PERIOD-INDEX PROBLEM}

Notation 3.2.1.7. The substack of semistable twisted sheaves is denoted by $\mathbf{T w}^{s s} \subset \mathbf{T w}$; the substack of stable sheaves is $\mathbf{T w}^{s} \subset \mathbf{T w}^{s s}$. The stack of semistable twisted sheaves with geometric Hilbert polynomial $P$ in the fibers is denoted by $\mathbf{T} \mathbf{w}_{\mathscr{X} / S}^{s s}(P)$.

The presence of the Brauer class of $\mathscr{X}$ can drastically simplify the meaning of (semi)stability, as in the following. We continue to assume that $S=\operatorname{Spec} k$ is the spectrum of a field. Let $\mathbf{T w} \mathbf{X}_{S}(n)$ temporarily denote the (open) substack parametrizing torsion free coherent $\mathscr{X}$-twisted sheaves of rank $n$.

Lemma 3.2.1.8. If $\mathscr{X} \rightarrow X$ is geometrically optimal, then the inclusion $\mathbf{T w}_{\mathscr{X} / S}^{S}(n) \hookrightarrow \mathbf{T w} \mathscr{X} / S(n)$ is an equality.

Proof. Given an $\mathscr{X}$-twisted sheaf $\mathscr{V}$ of rank $n$ on a geometric fiber of $X / S$, the fact that $\mathscr{X} \rightarrow X$ is geometrically optimal shows that any non-zero proper subsheaf $\mathscr{W} \subset \mathscr{V}$ must have full rank. (That is, $\mathscr{V} / \mathscr{W}$ is supported on a proper closed substack of $\mathscr{X}$.) In this case, the inequality required for stability follows immediately from the fact that a non-zero coherent $\mathscr{X}$-twisted sheaf has nonvanishing geometric Hilbert polynomial. (The reader is referred to [Lie07b, Lemma 2.2.7.13]; this seems to be a somewhat non-trivial fact, but it follows readily from the Riemann-Roch formula for representable morphisms of smooth Deligne-Mumford stacks with projective coarse spaces.)

Just as with ordinary sheaves, one can fix the determinant of a twisted sheaf. As described in Appendix A, there is a determinant morphism $\mathbf{T w}_{\mathscr{X} / S} \rightarrow \mathrm{Pic}_{\mathscr{X} / S}$ (more generally, if $X$ is not smooth one can only define this morphism on the locus parametrizing families whose fibers are perfect as objects in the derived category of coherent twisted sheaves). When the rank of $\mathscr{F}$ is $n$, det $\mathscr{F}$ is a 0 -fold twisted invertible sheaf (since $\mathscr{X}$ is a $\boldsymbol{\mu}_{n}$-gerbe), and is thus identified via pushforward with a section of $\mathrm{Pic}_{X / S}$. This defines a map $\mathbf{T w}_{\mathscr{X} / S}(n) \rightarrow \mathrm{Pic}_{X / S}$.

Definition 3.2.1.9. Given an invertible sheaf $\mathscr{L}$ on $X$ corresponding to $S \rightarrow \operatorname{Pic}_{X / S}$, the stack of twisted sheaves with determinant $\mathscr{L}$ is the stack-theoretic fiber product $\mathbf{T w} \mathbf{X}_{/ S}(n) \times_{\operatorname{Pic}_{X / S}} S$. The stack of semistable $\mathscr{X}$-twisted sheaves of rank $n$, geometric Hilbert polynomial $P$, and determinant $\mathscr{L}$ is denoted by $\mathbf{T w}_{\mathscr{X} / S}^{s s}(P, \mathscr{L})$, and similarly for stable objects.

Proposition 3.2.1.10. The stack $\mathbf{T} \mathbf{w}_{\mathscr{X} / S}^{s s}(P)$ has the following properties.

(i) It is an Artin stack of finite presentation over $S$.

(ii) It admits limits along any discrete valuation ring.

(iii) The open substack $\mathbf{T w}_{\mathscr{X} / S}^{S}(P)$ of stable twisted sheaves is a $\mathbf{G}_{m}$-gerbe over a separated algebraic space $\mathrm{Tw}_{\mathscr{X} / S}^{s}(P)$ of finite presentation over $S$.

(iv) If $X$ is a relative curve and $S$ is affine, then $\mathbf{T w}^{s s}$ is a GIT quotient stack, and is thus corepresented in the category of schemes by a projective scheme.

Given $\mathscr{L} \in \operatorname{Pic}_{X / S}(S)$, the stack $\mathbf{T w}_{\mathscr{X} / S}^{s s}(P, \mathscr{L})$ also satisfies (i), (ii), and (iv), and the open substack $\mathbf{T w}_{\mathscr{X} / S}^{s}(P, \mathscr{L})$ is a $\boldsymbol{\mu}_{n}$-gerbe over a separated algebraic space of finite presentation, hence is itself separated.

Remark 3.2.1.11. As in the case of ordinary sheaves, the Brauer class corresponding to the $\mathbf{G}_{m^{-}}$ gerbe $\mathbf{T w}^{s} \rightarrow \mathrm{Tw}^{s}$ is the famed Brauer obstruction to the existence of a tautological twisted sheaf on $\mathscr{X} \times \mathrm{Tw}^{s}$.

Remark 3.2.1.12. One can set up a similar theory of semistable coherent sheaves for any proper smooth Deligne-Mumford stack $\mathscr{X} \rightarrow S$ with projective coarse moduli space $X$ along the lines indicated above. From the point of view of period-index problems, this is particularly interesting when $\mathscr{X}$ is a $\boldsymbol{\mu}_{n}$-gerbe over an orbifold. This point will be pursued elsewhere. 


\section{LIEBLICH}

With these abstract foundations in hand, we describe how the theory specializes when $X$ is a curve or surface.

3.2.2 Moduli of twisted sheaves on curves. Fix a proper smooth curve $C$ over an algebraically closed field and a $\boldsymbol{\mu}_{n}$-gerbe $\pi: \mathscr{C} \rightarrow C$. It is a standard fact that $\mathrm{H}^{2}\left(C, \boldsymbol{\mu}_{n}\right)=\mathbb{Z} / n \mathbb{Z}$ (with a canonical generator being given by the first Chern class of the ideal sheaf of any closed point of $C$ ). Let $\bar{\delta}$ denote the (unique) fraction of the form $a / n$ such that $0 \leqslant a<n$ and $a \equiv[\mathscr{C}] \in \mathrm{H}^{2}\left(C, \boldsymbol{\mu}_{n}\right)$.

Definition 3.2.2.1. Given a sheaf $\mathscr{F}$ on $\mathscr{C}$, the degree of $\mathscr{F}$ is $n \operatorname{deg} c_{1}(\mathscr{F}) \in \mathbf{Q}$.

It is not hard to see that the denominator of the degree of $\mathscr{F}$ is in fact a factor of $n$ and that when $\mathscr{F}$ is pulled back from $C$, the degree computed above agrees with its degree as a sheaf on $C$. (Without the correcting factor of $n$, the denominator would be a factor of $n^{2}$, and degrees of pullbacks would be divided by $n$.)

Notation 3.2.2.2. We let $\mathbf{T w}_{\mathscr{C} / k}(r, d)$ denote the stack of twisted sheaves of rank $r$ and degree $d$ on $\mathscr{C}$. The superscripts s and ss are added to denote the stable or semistable locus as above. The coarse moduli space of $\mathbf{T} w^{\mathrm{s}}$ is written as $\mathrm{Tw}^{\mathrm{s}}$, as above. When the determinant is identified with $\mathscr{L}$, we write $\mathbf{T w}_{\mathscr{C} / k}(r, \mathscr{L})$ (with appropriate adornments for stable or semistable points).

We let $\mathbf{S h}_{C / k}(r, d)$ denote the stack of (untwisted) sheaves on $C$ with rank $r$ and degree $d$ and $\mathbf{S h}_{C / k}(r, L)$ denote the stack of sheaves with fixed determinant $L$. The (semi)stable locus is denoted with a superscript just as for twisted sheaves.

Proposition 3.2.2.3. There is a non-canonical isomorphism $\mathbf{T w}_{\mathscr{C} / k}(r, d) \stackrel{\sim}{\rightarrow} \mathbf{S h}_{C / k}(r, d-r \bar{\delta})$ which preserves the (semi)stable loci.

Sketch of proof. The key to the proof is the observation that any $\mathscr{C}$-twisted invertible sheaf has degree congruent to $\bar{\delta}$ modulo $\mathbb{Z}$. By Tsen's theorem, there is an invertible $\mathscr{C}$-twisted sheaf, say $\mathscr{T}$, of degree $\bar{\delta}$. Just as in the proof of Lemma 3.1.1.11, the functor $\mathscr{F} \mapsto \pi_{*}\left(\mathscr{F} \otimes \mathscr{T}^{\vee}\right)$ is an equivalence between the category of quasi-coherent $\mathscr{C}$-twisted sheaves and the category of quasi-coherent sheaves on $C$. Moreover, given a locally free $\mathscr{C}$-twisted sheaf $\mathscr{V}$, it is easy to see that $\mathscr{V}$ is semistable (as a twisted sheaf) if and only if $\pi_{*}\left(V \otimes \mathscr{T}^{\vee}\right.$ ) is semistable (as a locally free sheaf on $C$ ). Finally, if $\operatorname{deg} \mathscr{V}=d$, it follows from the usual formulas that $\operatorname{deg} \pi_{*}\left(\mathscr{V} \otimes \mathscr{T}^{\vee}\right)=\operatorname{deg}\left(\mathscr{V} \otimes \mathscr{T}^{\vee}\right)=d-r \bar{\delta}$, as required.

In particular, $\mathbf{T} \mathbf{w}_{\mathscr{C} / k}^{s s}(r, d)$ is a GIT stack (hence, corepresented by a projective variety).

The standard results concerning the moduli spaces of semistable sheaves on smooth curves carry over to the twisted setting. A relatively exhaustive list of references for these classical results may be found in [MFK94, Appendix 5C]. The final statement in the following uses the main result of [KS99], which was not available at the time [MFK94] was written.

Corollary 3.2.2.4. Assume that $C$ has genus at least two. Fix an invertible $r$-fold $\mathscr{C}$-twisted sheaf $\mathscr{L}$ of degree $d \in \mathbf{Q}$ (so that, in particular, $d-\bar{\delta} r \in \mathbb{Z}$ ).

(i) The stack $\mathbf{T w}_{\mathscr{C} / k}^{s s}(r, \mathscr{L})$ is smooth and is corepresented by a unirational projective variety $\mathrm{Tw}_{\mathscr{C} / k}^{s s}(r, \mathscr{L})$ of dimension $\left(r^{2}-1\right)(g-1)$. The map on stable loci $\mathbf{T w}_{\mathscr{C} / k}^{s}(r, \mathscr{L}) \rightarrow \mathrm{Tw}_{\mathscr{C} / k}^{s}(r, \mathscr{L})$ is naturally a $\boldsymbol{\mu}_{r}$-gerbe.

(ii) The stack $\mathbf{T w}_{\mathscr{C} / k}^{s s}(r, d)$ is integral and smooth over $k$. The stable locus is a $\mathbf{G}_{m}$-gerbe over an algebraic space of dimension $r^{2}(g-1)+1$.

(iii) If $d-r \bar{\delta}$ and $r$ are relatively prime, then the open immersion $\mathbf{T w}_{\mathscr{C} / k}^{s}(r, \mathscr{L}) \hookrightarrow \mathbf{T w}_{\mathscr{C} / k}^{s s}(r, \mathscr{L})$ is an isomorphism. In this case, $\operatorname{Tw}_{\mathscr{C} / k}^{s s}(r, \mathscr{L})$ is a smooth rational projective variety 


\section{TWISTED SHEAVES AND THE PERIOD-INDEX PROBLEM}

representing the sheafification of the stack $\mathbf{T w}_{\mathscr{C} / k}^{s s}(r, \mathscr{L})$. There is a tautological sheaf $\mathscr{F}$ on $\mathscr{C} \times \operatorname{Tw}_{\mathscr{C} / k}^{s s}(r, \mathscr{L})$, and $\operatorname{Pic}\left(\operatorname{Tw}_{\mathscr{C} / k}^{s s}(r, \mathscr{L})\right) \cong \mathbb{Z}$.

3.2.2.5 Now suppose that the base field $k$ is not necessarily algebraically closed, and assume that $n$ is invertible in $k$. Write $S=$ Spec $k$ and let $C \rightarrow S$ be a smooth projective geometrically connected curve with a section $p \in C(k)$. There is a way to describe the spaces $\operatorname{Tw}_{\mathscr{C} / k}^{s s}(r, d)$ using the theory of Galois twists. The Leray spectral sequence for $\mathbf{G}_{m}$ and the choice of $p$ yield an isomorphism $\mathrm{H}^{2}\left(C, \mathbf{G}_{m}\right)=\mathrm{H}^{2}\left(S, \mathbf{G}_{m}\right) \oplus \mathrm{H}^{1}\left(S, \mathrm{Pic}_{C / k}\right)$. Since $C$ has a point, it follows that $\mathrm{H}^{1}\left(S, \mathrm{Pic}_{C / k}\right)=$ $\mathrm{H}^{1}\left(S, \mathrm{Pic}_{C / k}^{0}\right)$. Similarly, there is a decomposition

$$
\mathrm{H}^{2}\left(C, \boldsymbol{\mu}_{n}\right)=\mathrm{H}^{2}\left(S, \boldsymbol{\mu}_{n}\right) \oplus \mathrm{H}^{1}\left(S, \operatorname{Pic}_{C / k}[n]\right) \oplus \mathrm{H}^{0}\left(S, \mathbf{R}^{2} f_{*} \boldsymbol{\mu}_{n}\right) .
$$

The sheaf $\mathbf{R}^{2} f_{*} \boldsymbol{\mu}_{n}$ is in fact isomorphic to $\mathbb{Z} / n \mathbb{Z}$, and a splitting of the natural map $\mathrm{H}^{2}\left(C, \boldsymbol{\mu}_{n}\right) \rightarrow$ $\mathrm{H}^{0}\left(S, \mathbf{R}^{2} f_{*} \boldsymbol{\mu}_{n}\right)=\mathbb{Z} / n \mathbb{Z}$ is given by sending $1 \in \mathbb{Z} / n \mathbb{Z}$ to the gerbe $[\mathscr{O}(p)]^{1 / n}$.

In particular, the gerbe $\mathscr{C}$ gives rise to an element $\tau \in \mathrm{H}^{1}\left(S, \mathrm{Pic}_{C / k}[n]\right)$ by projection. Note that tensoring yields a map $\operatorname{Pic}_{C / k}[n] \hookrightarrow \operatorname{Aut}\left(\operatorname{Sh}_{C \otimes \bar{k} / \bar{k}}^{s s}(n, \mathscr{O}(p))\right.$ ) (which is often an isomorphism [KP95]). By descent theory we see that varieties $V$ over $k$ which are geometrically isomorphic to $\operatorname{Sh}_{C / k}^{s s}(n, \mathscr{O}(p))$ are classified (up to isomorphism) by $\mathrm{H}^{1}\left(S, \operatorname{Aut}\left(\operatorname{Sh}_{C / k}^{s s}(n, \mathscr{O}(p))\right)\right)$. In particular, to any class $\tau \in \mathrm{H}^{1}\left(S, \mathrm{Pic}_{C / k}[n]\right)$ is associated a twist of $\operatorname{Sh}_{C / k}^{s s}(n, \mathscr{O}(p))$.

Proposition 3.2.2.6. With notation as above, let $0 \leqslant a<n$ correspond to the class of $\mathscr{C} \otimes \bar{k}$ in $\mathbb{Z} / n \mathbb{Z}=\mathrm{H}^{0}\left(S, \mathbf{R}^{2} f_{*} \boldsymbol{\mu}_{n}\right)$, and fix an invertible sheaf $\mathscr{M} \in \operatorname{Pic}(C)$. Then $\operatorname{Tw}_{\mathscr{C} / k}^{s s}(n, \mathscr{M})$ is the Galois twist of $\mathrm{Sh}_{C / k}^{s s}(n, \mathscr{M}(-a p))$ associated to the image of $[\mathscr{C}]$ in $\mathrm{H}^{1}\left(\mathrm{Spec} k, \mathrm{Pic}_{C / k}[n]\right)$ with respect to the decomposition of (1).

Sketch of proof. We first note that since $n$ is invertible in $k$ (by assumption), we see that the Brauer class of $\mathscr{C}$ will split over the separable closure of $k$, so that there is a $\mathscr{C} \otimes k^{\mathrm{sep}}$-twisted invertible sheaf $\mathscr{T}$ as in Proposition 3.2.2.3 such that $\mathscr{T}^{n} \cong \pi^{*} \mathscr{O}(a p)$ (so $\mathscr{T}$ has degree $a / n$ ). The proof now follows from the description of the isomorphism in Proposition 3.2.2.3 combined with an explicit cocycle computation.

In contrast to the classical case and Corollary 3.2.2.4(iii), the existence of a tautological family over $\operatorname{Tw}_{\mathscr{C} / k}^{s}\left(n, \mathscr{M}\right.$ ) (when $\operatorname{deg} \mathscr{M}-r$ is prime to $n$ ) is by no means assured. Since $\mathrm{Tw}^{s}$ is a smooth projective rational variety with Picard group $\mathbb{Z}$, it follows that the Brauer obstruction to the existence of a tautological sheaf is in fact the pullback of a Brauer class from the base field. As a consequence, we see, for example, that the existence of a tautological sheaf follows from the existence of a single stable twisted sheaf with the given invariants over the base field. (This in fact yields a slightly different proof that the classical Brauer obstruction vanishes in the untwisted case.) One way to interpret this is as follows: geometrically, one expects $\mathrm{Tw}^{s}$ to be quite similar to the space of vector bundles. The arithmetic of the twisting class is responsible for the more complex structure of the projection $\mathbf{T w}^{s} \rightarrow \mathrm{Tw}^{s}$. We return to this issue later when we study period-index problems for fibrations of curves.

3.2.3 Moduli of twisted sheaves on surfaces. Throughout this section, $\mathscr{X} \rightarrow X$ is a $\boldsymbol{\mu}_{n}$-gerbe on a smooth projective surface over a field $k$ and $n$ is assumed prime to char $(k)$. The two moduli spaces which are relevant are those coming from essentially trivial gerbes and from optimal gerbes. These should be thought of as complementary cases: given an Azumaya algebra $\mathscr{A}$ on $X$ with essentially trivial associated gerbe, one has that the generic fiber $\mathscr{A}(\eta)$ is simply $\mathrm{M}_{n}(k(X))$. If $\mathscr{A}$ has optimal associated gerbe, one has instead that $\mathscr{A}(\eta)$ is a division algebra over the function field $k(X)$. Twisted sheaves of rank $n$ on an optimal $\boldsymbol{\mu}_{n}$-gerbe can be thought of as objects in the 


\section{LIEBLICH}

'right non-commutative Picard space' of an integral non-commutative surface finite over its center. On the other hand, moduli of essentially trivial twisted sheaves are best understood in terms of a 'twisted stability condition' on the moduli of ordinary sheaves. This is described in detail in [Lie07b].

The stack of twisted sheaves with fixed rank $n$, determinant $L$, and geometric Hilbert polynomial $P$ is denoted by $\mathbf{T w}(n, L, P)$. Adornments, such as ${ }^{\mathrm{ss}}$, etc., are added as necessary. If instead we fix the pair $\left(L, c_{2}\right)$ of the determinant and degree of the second Chern class, we write $\mathbf{T w}\left(n, L, c_{2}\right)$. (These two ways of fixing the invariants are equivalent.) The superscript $\mu$ is used to denote the slope-stable locus, which is open in the Gieseker-stable locus.

Proposition 3.2.3.1. Suppose that $\mathscr{X}$ is geometrically essentially trivial. There is a non-canonical isomorphism

$$
\mathbf{T w}_{\mathscr{X} / k}^{\mu}(n, L, P) \otimes \bar{k} \stackrel{\sim}{\rightarrow} \mathbf{S h}_{X / k}^{\mu}\left(n, L^{\prime}, Q\right) \otimes \bar{k},
$$

with $Q$ an appropriate polynomial, where $\mathscr{X}=\left[L \otimes\left(L^{\prime}\right)^{\vee}\right]^{1 / n}$.

The proof is similar to the proof of Proposition 3.2.2.3.

Remark 3.2.3.2. In the above isomorphism, it is easy to see that the second Chern classes on the leftand right-hand sides differ by an amount which is bounded in terms of $L, \mathscr{X}$, and the intersection theory of $X$. Indeed, if $\mathscr{X} \otimes \bar{k}=[M]^{1 / n}$, then the isomorphism arises by sending $\mathscr{V}$ to $\mathscr{V} \otimes \mathscr{M}^{\vee}$, where $\mathscr{M}$ is an $\mathscr{X}$-twisted invertible sheaf such that $\mathscr{M}^{\otimes n} \cong M$. A standard computation shows that

$$
c_{2}\left(\mathscr{V} \otimes \mathscr{M}^{\vee}\right)=c_{2}(\mathscr{V})+(n-1) c_{1}\left(\mathscr{M}^{\vee}\right) c_{1}(\mathscr{V})+n\left(\begin{array}{l}
n \\
2
\end{array}\right) c_{1}\left(\mathscr{M}^{\vee}\right) .
$$

Thus, if $\operatorname{det} \mathscr{V}$ is fixed, then $c_{2}(\mathscr{V})$ and $c_{2}\left(\mathscr{V} \otimes \mathscr{M}^{\vee}\right)$ will differ by a fixed constant.

In the case of an optimal gerbe, we have the following theorem. The interested reader will find a proof along with proof of several other asymptotic properties (as $c_{2}$ grows) in [Lie07b].

Theorem 3.2.3.3. Suppose that $k$ is algebraically closed and $\mathscr{X}$ is optimal. Given an invertible sheaf $L \in \operatorname{Pic}(X)$, there is a constant $A$ such that for all $c_{2} \geqslant A$, the stack $\mathbf{T w}_{\mathscr{X} / k}^{s s}\left(n, L, c_{2}\right)$ is integral, normal, and local complete intersection whenever it is non-empty. Moreover, if $\mathbf{T w}_{\mathscr{X} / k}^{s s}\left(n, L, c_{2}\right)$ is non-empty, then $\mathbf{T} \mathbf{w}_{\mathscr{X} / k}^{s s}\left(n, L, c_{2}+2 r \ell\right)$ is non-empty for all $\ell \geqslant 0$.

Using the basic results from Appendix A on elementary transforms, we have the following nonemptiness result.

Proposition 3.2.3.4. Suppose that $\mathscr{X}$ is geometrically optimal. If there is a locally free $\mathscr{X}$ twisted sheaf of rank $n$, then for any integer $B$ and any invertible sheaf $L \in \operatorname{Pic}(X)$, the stack $\mathbf{T w}_{\mathscr{X} / k}^{s}\left(n, L, c_{2}\right)$ is non-empty for some $c_{2}>B$.

Proof. We may clearly assume that $k$ is algebraically closed. Since $\mathscr{X}$ is optimal, any torsion free $\mathscr{X}$-twisted sheaf of rank $n$ is automatically stable by Lemma 3.2.1.8. Moreover, if $\mathscr{F}$ is a torsion free $\mathscr{X}$-twisted sheaf of rank $n$ and $x \in X$ is a closed point, then the kernel $\mathscr{F}^{\prime}$ of any surjection $\mathscr{F} \rightarrow \mathscr{M}_{x}$ onto a $\mathscr{X} \times_{X} x$-twisted sheaf of rank one (a 'twisted skyscraper sheaf') has the same determinant as $\mathscr{F}$ and satisfies $c_{2}\left(\mathscr{F}^{\prime}\right)=c_{2}(\mathscr{F})+2 n$. Thus, it suffices to show that there is a locally free $\mathscr{X}$-twisted sheaf of rank $n$ and determinant $L$. Suppose that $\mathscr{V}$ is any locally free $\mathscr{X}$-twisted sheaf of rank $n$, and let $M=\operatorname{det} \mathscr{V}$. If $\mathscr{O}_{X}(1)$ is a very ample invertible sheaf, then there is some $n$ such that $L^{\vee} \otimes \operatorname{det}(\mathscr{V}(n))$ is very ample. Let $D \subset X$ be a smooth divisor in the linear system $\left|L^{\vee} \otimes \operatorname{det}(\mathscr{V}(n))\right|$. By Tsen's theorem, there is an invertible quotient of $\left.\mathscr{V}(n)\right|_{\mathscr{X} \times{ }_{X} D}$. Applying Corollary A.7 yields the result. 


\section{TWISTED SHEAVES AND THE PERIOD-INDEX PROBLEM}

We note that recent results of Langer [Lan06] on moduli of sheaves on arbitrary characteristic should give a method for proving Theorem 3.2.3.3 in the absence of the optimality hypothesis. In characteristic zero, it is likely that existing methods of O'Grady [Gra96] will carry over without much change to the twisted context, but we have not worked this out in detail.

Remark 3.2.3.5. For the geometrically minded, Theorem 3.2.3.3 plays an essential role in proving an algebraic analogue of results of Taubes on the stable topology of the space of self-dual connections on a fixed smooth $\mathrm{PGL}_{n}$-bundle over the 4-manifold underlying an algebraic surface. We refer such readers to $[\mathrm{Lie} 07 \mathrm{a}]$.

The same methods used in the classical case also yield information about the variation of the stack of stable twisted sheaves in a family. (The reader is referred to [Lie07b, Proposition 3.2.4.25] for a proof of the following.)

Proposition 3.2.3.6. Let $\mathscr{X} \rightarrow X \rightarrow S$ be a $\boldsymbol{\mu}_{n}$-gerbe on a smooth proper morphism over a locally Noetherian scheme with geometrically connected fibers of dimension two, and assume that $n$ is invertible on $S$. Suppose that for each geometric point $\bar{s} \rightarrow S$, the fiber $\mathscr{X}_{\bar{x}} \rightarrow X_{\bar{s}}$ is optimal or essentially trivial. Then the stack $\mathbf{T w}_{X / S}^{s}\left(n, L, c_{2}\right) \rightarrow S$ is a separated flat local complete intersection morphism for sufficiently large $c_{2}$.

We point out that the hypothesis on the geometric fibers of $\mathscr{X}$ is satisfied whenever $n$ is prime. This usually suffices for the study of period-index problems, as one can often reduce (by induction) to the case of classes of prime period.

\section{Period-index results}

\subsection{Preliminaries}

4.1.1 A cheap trick. We mention here a simple trick which can be used to make certain base extensions. For the sake of concreteness, we only record the result for field extensions.

Proposition 4.1.1.1. Let $K$ be a field and $\alpha \in \operatorname{Br}(K)$ a class annihilated by $n$. If $L / K$ is a finite field extension of degree $d$ and $n$ is relatively prime to $d$, then $\operatorname{per}(\alpha)=\operatorname{per}\left(\left.\alpha\right|_{L}\right)$ and $\operatorname{ind}(\alpha)=\operatorname{ind}\left(\left.\alpha\right|_{L}\right)$.

Proof. Write $X=\operatorname{Spec} K$ and $Y=\operatorname{Spec} L$. The equality of the periods follows immediately from the fact that the map $\mathrm{H}^{2}\left(X, \boldsymbol{\mu}_{n}\right) \rightarrow \mathrm{H}^{2}\left(Y, \boldsymbol{\mu}_{n}\right) \rightarrow \mathrm{H}^{2}\left(X, \boldsymbol{\mu}_{n}\right)$ induced by pullback and trace is multiplication by $d$. Suppose that $m$ is a positive integer with all prime factors dividing $n$. If there is a locally free $\alpha_{Y}$-twisted sheaf on $Y$ of rank $m$, then pushing it forward to $X$ yields a locally free $\alpha$-twisted sheaf of rank $m d$. Taking endomorphisms yields a central simple $K$-algebra $A$ of degree $m d$ with $[A]=\alpha$. If $D$ is the (unique up to isomorphism) central division $K$-algebra with class $\alpha$, we may write $A=\mathrm{M}_{r}(D)$ for some $r$. Since $\operatorname{per}(\alpha) \mid n$, we see that $(\operatorname{ind}(\alpha), d)=1$, so the degree of $D$ is prime to $d$. Since $m d=r \operatorname{deg} D$, we see that $d \mid r$. Thus, $m=\ell \operatorname{deg} D$ for some $\ell$, and we conclude that $\mathrm{M}_{\ell}(D)$ is a central simple algebra of degree $m$ with class $\alpha$, and thus that $\operatorname{ind}\left(\left.\alpha\right|_{L}\right) \geqslant \operatorname{ind}(\alpha)$. Since the reverse inequality is obvious, the result follows.

Corollary 4.1.1.2. If $X$ is a $k$-scheme and $\alpha \in \mathrm{H}^{2}\left(X, \mathbf{G}_{m}\right)$ has period $n$, then ind $(\alpha)=n$ if and only if there is a field extension $k^{\prime} \supset k$ of degree prime to $n$ such that $\operatorname{ind}\left(\alpha_{k^{\prime}}\right) \mid n$.

4.1.2 A reduction of geometric period-index problems to characteristic zero. Let $k$ be an algebraically closed field, $K / k$ a finitely generated field extension of finite transcendence degree and $\alpha \in \operatorname{Br}(K)$ a Brauer class. Let $W$ be the Witt ring of $k$; in particular, $W$ is an absolutely unramified Henselian discrete valuation ring with residue field $k$. 


\section{LIEBLICH}

Definition 4.1.2.1. Given a scheme $X$ and a point $x \in X$, an étale neighborhood of $x$ is an étale morphism $U \rightarrow X$ along with a lifting $x \rightarrow U$ over $x \rightarrow X$.

Proposition 4.1.2.2. With the above notation, there exists an extension of discrete valuation rings $W \subset R$ such that:

(i) $R$ is essentially of finite type and formally smooth over $W$;

(ii) there is an isomorphism $\rho$ between the residue field $\bar{R}$ and $K$;

(iii) for any finite extension $W \subset W^{\prime}$, the base change $R \otimes W^{\prime}$ is a discrete valuation ring with residue field isomorphic to $K$;

(iv) there is a class $\widetilde{\alpha} \in \operatorname{Br}(R)$ with the same period as $\alpha$ and such that $\left.\widetilde{\alpha}\right|_{\bar{R}}$ corresponds to $\alpha$ via $\rho$.

Proof. The idea of the proof is to first fiber a proper model of $K$ by curves over an affine space, use the deformation theory of curves to lift the function field over $W$, and then use the deformation theory of Azumaya algebras to lift the Brauer class over an étale localization of the global model.

First, we can choose a normal projective variety $X / k$ such that $k(X) \cong K$ (e.g., normalize the projective closure of an affine model!). By Bertini's theorem, a generic hyperplane section of $X$ will remain smooth in codimension one, so choosing a general pencil of very ample divisors on $X$, we can blow up $X$ to yield a birational model $\widetilde{X} \rightarrow \mathbf{P}^{1}$ with geometrically integral generic fiber of dimension $n-1$ which is smooth in codimension 1. By induction, it follows that we can find a smooth curve $C$ over the function field $k\left(\mathbf{P}^{n-1}\right)$ (with sections, if we like) such that the function field $K(C)$ is isomorphic to the original field $K$.

Now consider the local ring $A$ of $\mathbf{P}_{W}^{n-1}$ at the generic point $\eta$ of the special fiber $\mathbf{P}_{k}^{n-1}$. Over the residue field of $A$ we have a proper smooth curve $C$. Standard deformation theory (as may be found in [SGA1], for example) yields a proper smooth scheme $\mathfrak{C}$ over the formal completion $\widehat{A}$ with geometrically connected fibers of dimension one. By Artin approximation (or Popescu's theorem), there results such a scheme over the Henselization of $A$. The usual finite presentation arguments yield an étale neighborhood $U \rightarrow \mathbf{P}_{W}^{n-1}$ of $\eta$ and a proper smooth relative curve $\mathscr{C} \rightarrow U$ such that the fiber over $\eta$ is the original curve $C$. By shrinking $U$, we may assume that the fibers of $U$ over Spec $W$ are geometrically connected, hence that the fibers of $\mathscr{C}$ over $W$ are geometrically connected. The same follows for any étale neighborhood of the generic point $\gamma$ of the special fiber of $\mathscr{C}$ over $W$. Now $\mathscr{O}_{\mathscr{C}, \gamma}$ is a discrete valuation ring containing $W$ which satisfies all but possibly the last condition we require, and this remains true of any étale neighborhood of $\gamma$. Thus, the Henselization of $\mathscr{O}_{\mathscr{C}, \gamma}$ is the colimit of subrings quasi-finite over $\mathscr{O}_{\mathscr{C}, \gamma}$ satisfying conditions (i)-(iii). However, $\operatorname{Br}\left(\mathscr{O}_{\mathscr{C}, \gamma}^{h}\right)=\operatorname{Br}(K)$, so there is a lift of $\alpha$ to a class defined on $\mathscr{O}_{\mathscr{C}, \gamma}^{h}$, and this class descends to a class with the same period over some quasi-finite essentially finite type normal local extension $R$ of $\mathscr{O}_{\mathscr{C}, \gamma}$ with residue field $K$. This finishes the proof.

Given a domain $B$, we temporarily write $F(B)$ for its field of fractions.

Corollary 4.1.2.3. With the notation of Proposition 4.1.2.2, ind $\alpha=$ ind $\left.\widetilde{\alpha}\right|_{R \otimes_{W} \overline{F(W)}}$.

Proof. It is easy to see that there is a finite extension $W \subset W^{\prime}$ such that ind $\left.\widetilde{\alpha}\right|_{R \otimes_{W} F\left(W^{\prime}\right)}=$ ind $\left.\widetilde{\alpha}\right|_{R \otimes W} \overline{F(W)}$. However, $R \otimes W^{\prime}$ is a discrete valuation ring, so we can extend any division algebra over the fraction field $F\left(R \otimes W^{\prime}\right)=F(R) \otimes_{F(W)} F\left(W^{\prime}\right)$ to an Azumaya algebra over $R \otimes W^{\prime}$ with Brauer class $\left.\widetilde{\alpha}\right|_{W^{\prime}}$. Taking the residual algebra yields the result over the residue field of $R \otimes W^{\prime}$, which is $K$.

\subsection{Period and index on a fibration of curves}

4.2.1 Relation between period-index and the existence of rational points in moduli. 


\section{TWISTED SHEAVES AND THE PERIOD-INDEX PROBLEM}

Lemma 4.2.1.1. Let $X$ be a smooth geometrically connected projective variety over a field $k$ of dimension $d>0$. Suppose $X$ contains a 0-cycle of degree one (e.g. $k$ is finite). Given any prime number $n$, there is a finite separable extension $k^{\prime} \supset k$ of degree prime to $n$ and a birational equivalence of $X \otimes k^{\prime}$ with a fibration $\widetilde{X} \rightarrow \mathbf{P}_{k^{\prime}}^{d-1}$ with smooth generic fiber of dimension one and a rational point over $k^{\prime}\left(\mathbf{P}^{d-1}\right)$.

Proof. We may replace $k$ by the maximal separable prime to $n$ extension and suppose that $k$ is infinite and $X$ has a rational point $p \in X(k)$. Suppose first that $X$ is $\mathbf{P}^{d}$. Taking the linear system of hyperplanes through $p$, one sees that the blowup $\mathrm{Bl}_{p} \mathbf{P}^{d}$ fibers over $\mathbf{P}^{d-1}$ with a section and with generic fiber $\mathbf{P}_{k\left(\mathbf{P}^{d-1}\right)}^{1}$. Embedding $X$ in a projective space and choosing a generic linear projection yields a finite morphism $\nu: X \rightarrow \mathbf{P}^{d}$ which is étale at every point of $\nu^{-1}(\nu(p))$. Now we can simply pull back the picture from $\mathbf{P}^{d}$. The space $\widetilde{X}$ will then be the blowup of the fiber, which is just a finite set of reduced points. Geometrically, this is the same as taking a general linear system of projective dimension $d-1$ containing $p$ in the base locus in any sufficiently ample complete linear system on $X$.

Lemma 4.2.1.2. Let $X \leftarrow \widetilde{X} \rightarrow \mathbf{P}^{d-1}$ be as in Lemma 4.2.1.1. Given a class $\alpha \in \operatorname{Br}(X)$, one has

$$
\operatorname{ind}(\alpha)=\operatorname{ind}\left(\alpha_{\tilde{X}}\right)=\operatorname{ind}\left(\alpha_{\widetilde{X}_{\eta}}\right),
$$

where $\widetilde{X}_{\eta}$ is the generic fiber curve.

Proof. This follows from the fact that $\widetilde{X} \rightarrow X$ is birational and the index is determined at the generic point.

By construction, $\widetilde{X}_{\eta}$ has a rational point $p$.

Let $\pi: \widetilde{X}_{\eta} \rightarrow \eta=$ Spec $K$ be a proper smooth connected curve over a field with a rational point $p \in \widetilde{X}_{\eta}(K)$. Fix a class $\alpha \in \mathrm{H}^{2}\left(\widetilde{X}_{\eta}, \boldsymbol{\mu}_{n}\right)$ and let $0 \leqslant r<n$ be the class of $\left.\alpha\right|_{\bar{K}}$. Let $\mathscr{C} \rightarrow \widetilde{X}_{\eta}$ be a $\boldsymbol{\mu}_{n}$-gerbe representing $\alpha$.

Proposition 4.2.1.3. With the above notation, suppose that the restriction of $\alpha$ to $p$ vanishes in $\operatorname{Br}(\kappa(p))$. The following are equivalent:

(i) $\operatorname{per}(\alpha)=\operatorname{ind}(\alpha)$;

(ii) there is a locally free $\mathscr{C}$-twisted sheaf of rank $n$ and determinant of degree $r+1$.

If $n$ is prime and the $n$-cohomological dimension of $K$ is at most one, then (i) and (ii) are equivalent to the existence of a $K$-rational point on the coarse moduli space $\operatorname{Tw}_{\mathscr{C} / K}^{s}(n, \mathscr{O}((r+1) p))$, which is a smooth projective geometrically rational variety with Picard number one.

Proof. The equivalence of (i) and (ii) in the absence of the degree requirement is just Proposition 3.1.2.1(iii). Thus, the content of the proposition lies in adjusting the determinant. Suppose that there is a locally free twisted sheaf $\mathscr{F}$ of rank $n$ on $\mathscr{C}$, and let $\mathscr{M}=\operatorname{det} \mathscr{F}$. The assumption that $\left.\alpha\right|_{p}=0 \in \mathrm{H}^{2}\left(\operatorname{Spec} \kappa(p), \mathbf{G}_{m}\right)$ implies that there is a quotient of $\left.\mathscr{F}\right|_{p}$ with any rank at most $n$. Given such a quotient $Q$ of length $\ell$, it is easy to see that the determinant of the kernel $G$ of $\mathscr{F} \rightarrow Q$ is det $\mathscr{F}(-\ell p)$. (This is just an elementary transform; see Appendix A, for details.) By repeatedly applying this operation with various choices of $Q$ (and possibly dualizing to get the right sign), we can ensure that the determinant has degree $r+1$. (In fact, we can achieve any chosen degree in this way. The choice of $r+1$ is made clear below.) When $\operatorname{cd}_{n} K \leqslant 1$, we can use this argument at any effective Cartier divisor on $\widetilde{X}_{\eta}$ (as the $n$-torsion in the Brauer group of such a divisor vanishes) and therefore ensure that the determinant is precisely $\mathscr{O}((r+1) p)$. The stability condition when $n$ is prime can be ensured in one of two ways: (1) if the image $\widetilde{\alpha}$ of $\alpha$ in $\operatorname{Br}\left(\widetilde{X}_{\eta}\right)$ is zero, then it is a classical fact that stable vector bundles of any given rank and determinant exist; (2) if $\widetilde{\alpha} \neq 0$, then 


\section{LIEBLICH}

$\alpha$ is optimal, so any locally free twisted sheaf of rank $n$ is stable and thus geometrically stable by numerical considerations. (In general, only semistability is a geometric property, but in this case the two notions coincide.) Finally, the cohomological dimension hypothesis ensures that any point of the coarse space $\mathrm{Tw}_{\mathscr{C} / K}^{s}$ will lift into the stack $\mathbf{T w}_{\mathscr{C} / K}^{s}$, as the obstruction to doing so lies in $\operatorname{Br}(K)[n]$.

The fact that $\operatorname{Tw}_{\mathscr{C} / K}^{s}(n, \mathscr{O}((r+1) p)$ is smooth, projective, and rational with Picard number one follows from Proposition 3.2.2.3, the results cited above in $\S 3.2 .2$, and the main results of [KS99].

4.2.2 An application: period and index on a geometric surface. We give a proof of de Jong's theorem on the period-index problem for surfaces over algebraically closed fields.

Proposition 4.2.2.1. Let $K$ be a field of transcendence degree one over an algebraically closed field and $C \rightarrow \operatorname{Spec} K$ a smooth proper curve over $K$ with a rational point. Given a $\boldsymbol{\mu}_{n}$-gerbe $\mathscr{C} \rightarrow C$, let $0 \leqslant r<n$ represent the class of $\mathscr{C} \otimes \bar{K}$ in $\mathrm{H}^{2}\left(C \otimes \bar{K}, \boldsymbol{\mu}_{n}\right)=\mathbb{Z} / n \mathbb{Z}$. For any $\mathscr{L} \in \operatorname{Pic}(C)$ such that $\operatorname{deg} \mathscr{L}-r$ is relatively prime to $n$, there exists a stable locally free $\mathscr{C}$-twisted sheaf of rank $n$ and determinant $\mathscr{L}$.

Proof. Since any Brauer class is defined over a finitely generated field over the prime field, we immediately reduce to the case where $K$ is finitely generated over its constant field. By Corollary 3.2.2.4, $\operatorname{Tw}_{\mathscr{L} / K}^{s}(n, \mathscr{L}) \otimes \bar{K}$ is a non-empty smooth unirational projective variety. (We cite the unirationality rather than rationality as it is far easier to prove.) Applying the powerful theorem of Graber-Harris-Starr-de Jong (proven in [GHS03] in characteristic zero and generalized in [DS03] to positive characteristic), we conclude that $\operatorname{Tw}^{s}(n, \mathscr{L})$ has a rational point. On the other hand, $\mathbf{T w}^{s}(n, \mathscr{L}) \rightarrow \mathrm{Tw}^{s}(n, \mathscr{L})$ is a $\boldsymbol{\mu}_{n}$-gerbe. Since $K$ is $C_{1}$, any $\boldsymbol{\mu}_{n}$-gerbe over $K$ has a point, yielding a lift of the point in moduli to an object, as desired.

LEMma 4.2.2.2. If $X$ is a smooth surface over an algebraically closed field $k$ and $\alpha \in \operatorname{Br}(k(X))$ has order prime to $\operatorname{char}(k)$, then there is a birational morphism $\widetilde{X} \rightarrow X$ such that:

(i) the pullback of $\alpha$ to $\tilde{X}$ has strict normal crossing ramification locus, and

(ii) there is a fibration $\widetilde{X} \rightarrow \mathbf{P}^{1}$ with a section such that the restriction of $\alpha$ to the generic fiber is unramified.

Proof. It is an elementary fact that the ramification locus of $\alpha$ is pure of codimension one (see [AD03], [AM72], or [Sal97, Sal98]). Furthermore, if we blow up $X$, the only new ramification divisors which can appear must be the exceptional divisors. The first statement follows by the embedded resolution of curves in surfaces. To prove the second statement, we note that the ramification of $\alpha$ at a divisor $D$ is given by a cyclic extension of the function field $\kappa(D)$ (see [AM72, Sal97, Sal98]). (In fact, a generically separable extension is sufficient for the present argument, and this follows easily from basic calculations in étale cohomology [Gro68a] applied to the usual presentation for the sheaf of Cartier divisors.) Let $E$ be the (normal crossing) ramification divisor of $\alpha$. Choose a divisor $Z$ such that $Z$ intersects $E$ transversely at general points of irreducible components and $Z+E$ is very ample. Choose a section $H$ of $|Z+E|$ intersecting $Z$ transversely and intersecting $E$ transversely at general points of irreducible components over which the cyclic extensions measuring ramification are unramified. It follows by an explicit cohomological calculation (or by the slightly more hands-on method of [Sal97, Sal98]) that the blowup of $X$ at $H \cap(Z+E)$ has no ramification on the exceptional divisors, i.e. the ramification of $\alpha$ on $\widetilde{X}$ is precisely contained in $E$. This $\widetilde{X}$ is the total space of the pencil spanned by $H$ and $Z+E$, and we have just seen that $\alpha$ is unramified on the generic fiber. The intersection points $H \cap(Z+E)$ give rise to sections of the pencil.

Theorem 4.2.2.3 (De Jong without restrictions). If $K$ is a field of transcendence degree two over an algebraically closed field $k$, then any $\alpha \in \operatorname{Br}(K)$ satisfies $\operatorname{per}(\alpha)=\operatorname{ind}(\alpha)$. 


\section{TWISTED SHEAVES AND THE PERIOD-INDEX PROBLEM}

Proof. As above, we may assume that $K$ is finitely generated over $k$. By the results of $\S 4.1 .2$, we may assume the base field has characteristic zero. (The reader can check that this proof in fact works whenever the period is prime to the characteristic exponent of the base field.) Let $X$ be a smooth projective surface modeling the given function field and let $\alpha \in \operatorname{Br}(k(X))[n]$. Applying Lemma 4.2.2.2, we may replace $X$ with a fibration $\widetilde{X} \rightarrow \mathbf{P}^{1}$ such that $\alpha$ is unramified on the generic fiber. Now we can apply Proposition 4.2.2.1. (The reader unfamiliar or uncomfortable with the ramification of a Brauer class can simply consider unramified classes; these will suffice for the applications we give over finite fields, at the expense of generality.)

Elementary transforms can be used to refine Theorem 4.2.2.3.

Corollary 4.2.2.4. Let $X$ be a smooth projective surface over an algebraically closed field and $n$ an integer prime to char $(X)$. The natural map $\mathrm{H}^{1}\left(X, \mathrm{PGL}_{n}\right) \rightarrow \mathrm{H}^{2}\left(X, \boldsymbol{\mu}_{n}\right)$ is surjective.

Proof. Given a $\boldsymbol{\mu}_{n}$-gerbe $\mathscr{X} \rightarrow X$, Proposition 4.2.2.1 shows that there is a locally free (stable) $\mathscr{X}$-twisted sheaf $\mathscr{V}$ of rank $n$. Applying the methods used in the proof of Proposition 3.2.3.4 yields a $\mathscr{V}$ with trivial determinant. Taking $\mathscr{E} n d(\mathscr{V})$ yields an Azumaya algebra whose cohomology class in $\mathrm{H}^{2}\left(X, \boldsymbol{\mu}_{n}\right)$ is precisely [ $\mathscr{X}$ ] (see Proposition 3.1.2.1(iv)).

Remark 4.2.2.5. One can also prove Corollary 4.2.2.4 for classes of order divisible by the characteristic (using the fppf topology to compute the cohomology) by applying the methods of Proposition 3.2.3.4 to fppf $\boldsymbol{\mu}_{n}$-gerbes on the surface $X$.

4.2.3 A local-to-global problem. A consequence of twisted methods is that one can solve the period-index problem in general if one can characterize the points on Galois twists of the moduli space $\operatorname{Sh}_{C / k}^{s}(r, \mathscr{L})$ of stable vector bundles with fixed determinant of degree prime to the rank on a smooth curve $C$ with a point. Much is known about these varieties: they are smooth and rational with Picard group $\mathbb{Z}$. Moreover, one knows that the canonical class of $\operatorname{Sh}_{C / k}^{s}(r, \mathscr{L})$ is twice an anti-ample generator (so that $\mathrm{Sh}^{s}$ is not a projective space) [Ram73].

It is tempting to ask the following question.

Question 4.2.3.1. Let $k$ be a field and $C / k$ a smooth projective curve with a rational point. Given an invertible sheaf $\mathscr{L} \in \operatorname{Pic}(C)$ and an integer $n$ prime to $\operatorname{deg} \mathscr{L}$, does any Galois twist of $\operatorname{Sh}_{C / k}^{s}(n, \mathscr{L})$ coming from $\mathrm{H}^{1}\left(\operatorname{Spec} k, \operatorname{Pic}_{C / k}^{0}[n]\right)$ have a $k$-rational point?

For a general curve $C / k$, the automorphism group of $\operatorname{Sh}_{C / k}^{s}(n, \mathscr{L})$ is precisely $\operatorname{Pic}_{C / k}^{0}[n]$, so the question is asking whether in such cases, any form of $\operatorname{Sh}_{C / k}^{s}(n, \mathscr{L})$ has a rational point. This is closely related to the period-index problem in the following way.

Proposition 4.2.3.2. An affirmative answer to Question 4.2.3.1 implies that for any function field $K$ of dimension $d$ with algebraically closed constant field $k$ and any Brauer class $\alpha \in \operatorname{Br}(K)$, we have ind $(\alpha) \mid \operatorname{per}(\alpha)^{2}$.

Sketch of a proof. Let $n=\operatorname{per}(\alpha)$. Given $K=k(X)$, we can birationally fiber $X$ over $\mathbf{P}^{d-1}$ such that:

(i) the generic fiber $C / k\left(x_{1}, \ldots, x_{d-1}\right)$ is smooth with a section; and

(ii) $\alpha$ is unramified on the generic fiber and trivial on the section.

(Such a fibration comes from an appropriate linear system of hyperplane sections, and the section arises from a base point of the system.) For an appropriate choice of $\mathscr{L}$, the coarse moduli space $\operatorname{Tw}^{s}(n, \mathscr{L})$ has a rational point by Proposition 3.2.2.6 and Question 4.2.3.1. We have the following lemma. 


\section{LIEBLICH}

Lemma 4.2.3.3. Let $C / k$ be a proper smooth connected curve with a rational point $p$ and $\mathscr{C} \rightarrow C$ a $\boldsymbol{\mu}_{n}$-gerbe such that $\mathscr{C} \times_{C} p$ is trivial. For any point $q \in \mathrm{Tw}_{\mathscr{C} / k}^{s}$, the index of the class in $\operatorname{Br}(\kappa(q))$ represented by $\mathbf{T w}_{\mathscr{C} / k}^{s} \times{ }_{\mathrm{Tw}_{\mathscr{C} / k}^{s}}^{s} q$ divides $n$.

Proof. Let $\mathscr{F}$ be the universal $\mathscr{C}$-twisted sheaf on $\mathscr{C} \times_{k} \mathbf{T w}_{\mathscr{C} / k}^{s}$. The universality of $\mathscr{F}$ implies that for any point $p \rightarrow \mathscr{C}$, the pullback $\mathscr{F} p$ is $\mathbf{T w}_{\mathscr{C} / k}^{s}$-twisted. Thus, choosing $p: \operatorname{Spec} k \rightarrow \mathscr{C}$ splitting the restriction $\mathscr{C} \times_{C} p$ yields a locally free $\mathbf{T w}_{\mathscr{C} / k}^{s}$-twisted sheaf of rank $n$, as desired.

Thus, the obstruction to lifting the point into $\mathbf{T} \mathbf{w}^{s}$ has index dividing $n$, so that, after making a base field extension of degree $n$, there is a locally free twisted sheaf of rank $n$. Pushing this sheaf forward yields the result.

On the other hand, Gabber's appendix to [Col02] (answering a question of Colliot-Thélène in the affirmative) shows that for a fixed prime $\ell$ and any positive integer $d$, there is a smooth projective $d$-fold $X$ over $\mathbb{C}$ and an unramified Brauer class $\alpha \in \operatorname{Br}(X)$ such that $\operatorname{per}(\alpha)=\ell$ and ind $(\alpha)=\ell^{d-1}$. Taking a generic fibration of $X$ as in Proposition 4.2.3.2, we conclude the following.

Corollary 4.2.3.4. Given a prime $\ell$ and a positive integer $d$, there is a smooth proper connected curve $C / \mathbb{C}\left(t_{1}, \ldots, t_{d-1}\right)$ with a section and a $\boldsymbol{\mu}_{\ell}$-gerbe $\mathscr{C} \rightarrow C$ such that for every $L \in \operatorname{Pic}(C)$, the coarse moduli space $\mathrm{Tw}_{\mathscr{C} / k}^{s}(\ell, L)$ has the property that any closed point has degree divisible by $\ell^{d-3}$. Moreover, $\mathscr{C} \rightarrow C$ may be chosen so that its associated Brauer class is unramified on a proper smooth model of $C$ over $\mathbb{C}$.

Proof. We can fiber one of Gabber's examples to yield $\mathscr{C} \rightarrow C$ representing a Brauer class of period $\ell$ and index $\ell^{d-1}$. (The ramification condition comes from the fact that the Brauer group is a birational invariant and Gabber's examples begin life as unramified classes.) If there is a closed point whose degree has $\ell$-adic valuation smaller than $d-3$, then there is a locally free $\mathscr{C}$-twisted sheaf whose rank has $\ell$-adic valuation smaller than $d-1$. (One additional factor of $\ell$ is potentially needed to lift the point into the moduli stack as in Lemma 4.2.3.3, and the other factor of $\ell$ comes from the fact that the stack parametrizes $\mathscr{C}$-twisted sheaves of rank $\ell$.) However, this contradicts the fact that the index is $\ell^{d-1}$ (using Corollary 4.1.1.2).

There is a somewhat amusing consequence of this fact for local-to-global problems over twodimensional function fields.

Proposition 4.2.3.5. For any positive integer $N$, there is a variety $V$ over $\mathbb{C}\left(t_{1}, t_{2}\right)$ such that:

(i) $\operatorname{dim} V>N$;

(ii) $V$ is smooth, projective, geometrically connected, and geometrically rational;

(iii) $\operatorname{Pic}\left(V \otimes \overline{\mathbb{C}\left(t_{1}, t_{2}\right)}\right) \cong \mathbb{Z}$;

(iv) $V\left(\mathbb{C}\left(t_{1}, t_{2}\right)_{\nu}\right) \neq \emptyset$ for every non-trivial valuation $\nu$ on $\mathbb{C}\left(t_{1}, t_{2}\right)$, but

(v) $V\left(\mathbb{C}\left(t_{1}, t_{2}\right)\right)=\emptyset$.

Sketch of proof. Choose $\mathscr{C} \rightarrow C$ as in Corollary 4.2.3.4 with $d=3$, so that $C$ is the generic fiber of a proper fibration $\mathcal{C} \rightarrow \mathbf{P}_{\mathbb{C}}^{2}$ and $\mathscr{C}$ is the restriction of a $\boldsymbol{\mu}_{n}$-gerbe over $\mathcal{C}$. Choose $L$ so that it is the restriction of an invertible sheaf on $\mathcal{C}$ and so that $\operatorname{deg} L-\ell \bar{\delta}$ is relatively prime to $\ell$, where $\bar{\delta}$ is as in $\S 3.2 .2$. (After birationally modifying the base, we may assume that any given $L \in \operatorname{Pic}(C)$ extends, without doing any harm to the argument given here.) Given a non-trivial valuation $\nu$ of $\mathbb{C}\left(t_{1}, t_{2}\right)$, the valuation ring $\mathscr{O}_{\nu}$ will be centered at a point $p_{\nu}$ of $\mathbf{P}_{\mathbb{C}}^{2}$ whose residue field has transcendence degree at most one. The fiber $\mathcal{C}_{p_{\nu}}$ is a curve over the function field of a curve or over an algebraically closed field, so we may apply the methods of Proposition 4.2.1.3 to find a locally free $\mathscr{C}_{p_{\nu}}$-twisted sheaf of rank $\ell$ with determinant $L_{p_{\nu}}$. (When the fiber is not smooth, we can use the unobstructedness 


\section{TWISTED SHEAVES AND THE PERIOD-INDEX PROBLEM}

of locally free sheaves on curves to see that it suffices to solve the problem for the reduced structure on the fiber, then apply Proposition 4.2.1.3 as written to the normalization and produce a twisted sheaf over the singular space by a gluing argument.) It is a simple matter of deformation theory to see that this will deform over the complete local ring $\widehat{\mathscr{O}}_{\mathbf{P}^{2}, p_{\nu}}$. Pulling back to the completion of $\mathbb{C}\left(t_{1}, t_{2}\right)$ with respect to $\nu$ yields a locally free $\mathscr{C} \otimes \mathbb{C}\left(t_{1}, t_{2}\right)_{\nu}$-twisted sheaf of rank $\ell$ and determinant $L$, which we may assume is stable (by a simple argument as in the proof of Proposition 4.2.1.3). The reader can find further details of this kind of argument and applications of this method in more complicated (e.g. ramified) situations in [Lie06].

On the other hand, a simple argument using the Leray spectral sequence shows that the map $\operatorname{Br}\left(\mathbb{C}\left(t_{1}, t_{2}\right)\right) \rightarrow \operatorname{Br}\left(\operatorname{Tw}_{\mathscr{C} / \mathbb{C}\left(t_{1}, t_{2}\right)}^{s}(\ell, L)\right)$ is an isomorphism. It follows from the previous paragraph that the universal obstruction $\mathbf{T w}_{\mathscr{C} / \mathbb{C}\left(t_{1}, t_{2}\right)}^{s}(\ell, L) \rightarrow \mathrm{Tw}_{\mathscr{C} / \mathbb{C}\left(t_{1}, t_{2}\right)}^{s}(\ell, L)$ is split over every completion of $\mathbb{C}\left(t_{1}, t_{2}\right)$. However, any element of $\operatorname{Br}\left(\mathbb{C}\left(t_{1}, t_{2}\right)\right)$ which is locally split everywhere must be split, and thus a $\mathbb{C}\left(t_{1}, t_{2}\right)$-rational point of $\operatorname{Tw}_{\mathscr{C} / \mathbb{C}\left(t_{1}, t_{2}\right)}^{s}(\ell, L)$ would give rise to a $\mathscr{C}$-twisted sheaf of rank $\ell$, contradicting the fact that Brauer class of $\mathscr{C}$ has index $\ell^{2}$.

Remark 4.2.3.6. The fact that the base field in Proposition 4.2.3.5 is $\mathbb{C}\left(t_{1}, t_{2}\right)$ is significant, because its unramified Brauer group is trivial. It is quite a bit easier to make such examples over other function fields of transcendence degree two: if $P \rightarrow S$ is a Brauer-Severi scheme representing a non-trivial Brauer class over a proper smooth surface $S$, then it is easy to see that $P$ satisfies all of the conditions of Proposition 4.2.3.5.

Remark 4.2.3.7. Using [Col02, Théorème 10], one can make the counterexamples $V$ of Corollary 4.2.3.4 be defined over $k\left(t_{1}, t_{2}\right)$ with $k$ any algebraically closed field.

\subsection{Period and index via moduli on a surface}

Another way to reduce the period-index problem to a rationality question is to use the known structure of the moduli spaces of twisted sheaves on surfaces along with classical estimates on the existence of points (e.g. the Lang-Weil estimates for geometrically integral varieties over finite fields).

\subsubsection{Period and index on a surface over a finite field.}

Theorem 4.3.1.1. If $X$ is a proper smooth geometrically connected surface over $\mathbf{F}_{q}$ and $\alpha \in \operatorname{Br}(X)$ has period prime to $q$, then $\operatorname{per}(\alpha)=\operatorname{ind}(\alpha)$.

For classes of period divisible by the characteristic, the methods employed here cannot be applied (even though the moduli theory can be developed). Thus, (sadly) we can give no real insight into the unramified problem for these classes.

Remark 4.3.1.2. If $K$ is the function field of a surface over a finite field of characteristic $p$, then it is easy to see that for $\alpha \in \operatorname{Br}(K)\left[p^{\infty}\right]$ we have $\operatorname{ind}(\alpha) \mid \operatorname{per}(\alpha)^{2}$. Indeed, the absolute Frobenius morphism $F: \operatorname{Spec} K \rightarrow \operatorname{Spec} K$ is finite free of degree $p^{2}$ and acts as multiplication by $p$ on $\operatorname{Br}(K)$. Curiously enough, this result is stronger than what we are able to prove in $\S 4.3 .2$ for ramified classes of period prime to the characteristic.

Remark 4.3.1.3. We thank Laurent Moret-Bailly for pointing out that the proof of Theorem 4.3.1.1 actually works for Brauer classes on a proper smooth geometrically connected surface over any perfect field $k$ with the property that any geometrically integral finite type $k$-scheme contains a 0 -cycle of degree one. For example, the theorem applies to any PAC base field. (Using Proposition 4.3.1.6, whose proof we have not included here, would allow one to remove the perfect hypothesis in the preceding sentence.) 


\section{LIEBLICH}

Owing to the inadequacy of the existing proofs of asymptotic properties [Lie07b] (e.g. applying only to geometrically optimal classes and geometrically essentially trivial classes), we are forced to make a slightly convoluted argument. Let $\operatorname{PIP}_{n}(k)$ denote the phrase "classes $\alpha$ of period dividing $n$ in the Brauer group of any geometrically connected proper smooth surface over $k$ satisfy $\operatorname{per}(\alpha)=$ $\operatorname{ind}(\alpha)^{\prime}$.

Proposition 4.3.1.4. If $k$ is a perfect field and $P I P_{\ell}(L)$ for all finite extensions $L / k$ and all primes $\ell$ in a set of primes $S$, then $\operatorname{PIP}_{n}(L)$ for all finite extensions $L$ and all $n$ in the submonoid $M$ of $\mathbf{N}$ generated by $S$.

Proof. Let $\alpha \in \operatorname{Br}(X)$ have period $n \in M$. As above, we see that $n^{\prime} \alpha$ has the period equal to index (where $n^{\prime}=n / \ell$ for some prime factor $\ell$ of $n$ ). Thus, $n^{\prime} \alpha$ has index $\ell$. Since any division algebra over a field of index $d$ has a separable splitting field of degree $d$, we conclude that there is a generically étale finite map of normal surfaces $f: Y \rightarrow X$ of degree $\ell$ such that $f^{*} n^{\prime} \alpha=0$. By the resolution of singularities in dimension two (valid in any characteristic), we arrive at a regular proper surface $\widetilde{Y}$ and a morphism $\widetilde{Y} \rightarrow X$ which is generically finite of degree $\ell$. Since $k$ is perfect, we see that $\widetilde{Y}$ is smooth over $k$; in fact, setting $L=\mathrm{H}^{0}\left(Y, \mathscr{O}_{Y}\right)$, we see that $\widetilde{Y}$ is smooth, proper, and geometrically connected over $L$. Applying $P I P_{n^{\prime}}(L)$ to $Y$ and pushing forward at the generic point completes the proof.

Corollary 4.3.1.5. To prove Theorem 4.3.1.1 it suffices to prove it for classes of prime order (prime to q). Thus, it suffices to prove it for classes which are either geometrically optimal or geometrically essentially trivial.

Using results of Artin and de Jong on general sections of an Azumaya algebra, one can actually prove a stronger form of Proposition 4.3.1.4. Since it is not essential for our applications, we only record the statement.

Proposition 4.3.1.6. If $k$ is any field and $P I P_{\ell}(k)$ for all primes $\ell$ in a set of primes $S$, then $P I P_{n}(k)$ for all $n$ in the submonoid of $\mathbb{N}$ generated by $S$.

Proof of Theorem 4.3.1.1 for geometrically optimal classes. Let $\mathscr{X} \rightarrow X$ be a geometrically optimal $\boldsymbol{\mu}_{n}$-gerbe. Consider the stacks $\mathbf{T w}_{\mathscr{X} / \mathbf{F}_{q}}^{s}(n, \mathscr{O}, b)$, which are defined over $\mathbf{F}_{q}$ since $\mathscr{O}$ is defined over $\mathbf{F}_{q}$. By Theorem 3.2.3.3 and Proposition 3.2.3.4 (applied over $\overline{\mathbf{F}}_{q}$, using Theorem 4.2.2.3 as an input), there exists $b$ such that $\mathbf{T} \mathbf{w}_{\mathscr{X} / \mathbf{F}_{q}}^{s}(n, \mathscr{O}, b)$ is (non-empty and) geometrically integral. Thus, $\operatorname{Tw}_{\mathscr{X} / \mathbf{F}_{q}}^{s}(n, \mathscr{O}, b)$ is a geometrically irreducible (even projective) variety over $\mathbf{F}_{q}$, and furthermore since $\mathrm{H}^{2}\left(\operatorname{Spec} \mathbf{F}_{q}, \boldsymbol{\mu}_{n}\right)=0$, we see that a rational point of $\operatorname{Tw}_{\mathscr{X} / \mathbf{F}_{q}}^{s}(n, \mathscr{O}, b)$ lifts to an object of $\mathbf{T w}_{\mathscr{X} / \mathbf{F}_{q}}^{s}\left(n, \mathscr{O}, c_{2}\right)$. Thus, by the cheap trick (§4.1.1), it suffices to find a rational point of $\operatorname{Tw}_{\mathscr{X} / \mathbf{F}_{q}}^{s}(n, \mathscr{O}, b)$ over $\mathbf{F}_{q}^{\text {non- } n}$, the maximal extension of degree prime to $n$. However, the LangWeil estimates [LW54] show that any non-empty geometrically integral variety over $\mathbf{F}_{q}$ has rational points over $\mathbf{F}_{q}^{\text {non- } n}$.

Proof of Theorem 4.3.1.1 for geometrically essentially trivial classes. In this case $\mathscr{X} \rightarrow X$ is a $\boldsymbol{\mu}_{n^{-}}$ gerbe such that $\mathscr{X} \otimes \overline{\mathbf{F}}_{q}$ admits an invertible twisted sheaf (has trivial Brauer class). By Proposition 3.2.3.1, the stack $\mathbf{T} \mathbf{w}^{\mu}\left(n, \mathscr{O}, c_{2}\right)$ is geometrically isomorphic to $\mathbf{S h}^{\mu}\left(n, \mathscr{L}, c_{2}^{\prime}\right)$ for some $\mathscr{L}$ (possibly defined only over an extension field, but this is unimportant) and a $c_{2}^{\prime}$ which differs from $c_{2}$ by a fixed constant depending only on $\mathscr{X}$ by Remark 3.2.3.2. On the other hand, the stack of semistable sheaves on $X$ is asymptotically geometrically irreducible and non-empty (in the sense of the statements of Theorem 3.2.3.3 and Proposition 3.2.3.4), with $\mathbf{S} \mathbf{S h}^{\mu}$ as a dense open substack. The proof in arbitrary characteristic (generalizing O'Grady's results in characteristic zero) is contained in [Lan06]. Applying the Lang-Weil estimates to the substack of $\mu$-stable points of $\mathbf{T} \mathbf{w}^{s}$ completes the proof. 


\section{TWISTED SHEAVES AND THE PERIOD-INDEX PROBLEM}

4.3.2 A ramified result. Using results of Saltman [Sal97, Sal98], we can extend our results to the ramified case. We recall the relevant results from Saltman's work (and we thank him for bringing this to our attention).

Proposition 4.3.2.1 (Saltman). Let $S$ be a regular scheme of dimension two fibered over a field or discrete valuation ring containing a primitive $\ell$ th root of unity, with $\ell$ a prime invertible on $S$. Let $\alpha$ be a Brauer class at the generic point $\eta_{S}$ of prime order $\ell$. There exist rational functions $f, g \in \kappa(S)$ such that $\alpha$ is unramified in $\kappa(S)\left(f^{1 / \ell}, g^{1 / \ell}\right)$.

Saltman uses this to prove that ind $\mid$ per $^{2}$ for Brauer classes in the function field of a curve over a $p$-adic field. Note that the requirement that an $\ell$ th root of unity exist is minor: adjoining such a root creates a field extension of degree prime to $\ell$, so using the methods of $\S 4.1 .1$ one concludes that this has no effect on the period-index problem. Similarly, the requirement that $\alpha$ have prime order is immaterial when it comes to period-index phenomena.

COROLlary 4.3.2.2. Let $X$ be a smooth projective geometrically connected surface over a finite field $k$. Any $\alpha \in \operatorname{Br}(\kappa(X))$ satisfies $\operatorname{ind}(\alpha) \mid \operatorname{per}(\alpha)^{3}$.

Proof. By Remark 4.3.1.2, it suffices to prove this when the period of $\alpha$ is a prime $\ell$, and then we may assume that $k$ contains all of the $\ell$ th roots of unity by making a prime to $\ell$ extension of the base field (which will not change the period or index by Proposition 4.1.1.1). In this case, we can apply Proposition 4.3.2.1 to find a finite extension of normal surfaces $Y \rightarrow X$ of degree $\ell^{2}$ such that $\left.\alpha\right|_{Y}$ is unramified. Letting $L=\mathrm{H}^{0}\left(Y, \mathscr{O}_{Y}\right)$, we can blow up $Y$ to arrive at a smooth geometrically connected surface $\widetilde{Y}$ over $L$ equipped with a Brauer class $\alpha \in \operatorname{Br}(\widetilde{Y})$ of order dividing $\ell$. Since $Y$ is proper, $L$ is finite, and we may apply Theorem 4.3.1.1 to conclude that the index of $\alpha_{\widetilde{Y}}$ divides $\ell$. Pushing forward a twisted sheaf of rank dividing $\ell$ along the morphism of generic points $\eta_{Y} \rightarrow \eta_{X}$ yields an $\alpha$-twisted sheaf of rank dividing $\ell^{3}$ on $\operatorname{Spec} k(X)$ and taking the endomorphism ring yields a division algebra of degree dividing $\ell^{3}$ in the class $\alpha$, thus completing the proof.

4.3.3 Period and index on a surface over a local field. Using Theorem 4.3.1.1 and Proposition 3.2.3.6, we can prove a partial result on the period-index problem for surfaces over local fields. Throughout this section, $K$ denotes a local field with integer ring $R$ and (finite) residue field $k$.

Proposition 4.3.3.1. Let $X$ be a proper smooth geometrically connected surface over $K$ which extends to a proper smooth relative surface $\mathcal{X} \rightarrow \operatorname{Spec} R$. If $\alpha \in \operatorname{Br}(X)$ has period prime to char $(k)$, then $\operatorname{ind}(\alpha) \mid \operatorname{per}(\alpha)^{2}$. If $\alpha$ is unramified on $\mathcal{X}$, then $\operatorname{ind}(\alpha)=\operatorname{per}(\alpha)$.

Proof. First suppose that $\alpha$ extends to all of $\mathcal{X}$. On the special fiber, we have that per $=$ ind. It is not too difficult to show that one can construct an unobstructed locally free twisted sheaf of the desired rank using strict transforms (see, e.g., [HL97, Theorem 5.2.5 and Remark 5.2.6] for the untwisted version, [Lie04] for the twisted version, or [Dej04, Proposition 3.2] for a treatment in terms of Azumaya algebras). Deforming it over the total space of $\mathcal{X}$ yields the result.

If $\alpha$ is ramified along the special fiber, then extracting the $\operatorname{per}(\alpha)$ th root of a uniformizer of $R$ will kill the ramification [AD03, Theorem 2.3.4] (which uses the main purity result of [Gab81]). Thus, after making a finite free extension $R^{\prime} \supset R$ of degree $\operatorname{per}(\alpha)$, we are reduced to the unramified case. This is easily seen to imply the desired result.

It would be nice to try to apply Saltman's techniques to the case of local base fields $k$ and arrive at a result for ramified classes, but it is not entirely clear how the property of smooth reduction behaves under the construction of $\widetilde{Y}$ in the proof of Corollary 4.3.2.2.

Remark 4.3.3.2. It may be possible to extend these results in some form to the case of a surface with semistable or strictly semistable reduction. To properly carry this out would require a study of 


\section{LIEBLICH}

semistable twisted sheaves and their deformations on semistable surfaces and their (possibly nonflat) infinitesimal thickenings. It is not particularly clear at the present time what exponent in the period-index relation one should expect in this case.

\section{Appendix A. A few facts about coherent sheaves on stacks}

There is not much to recall here, as coherent sheaves behave just as they do on schemes. However, for the sake of completeness, we include stacky versions of several well-known constructions and results. First, it is worth making a brief remark about where precisely quasi-coherent sheaves on a stack $\mathscr{X}$ live. Of course, they are quasi-coherent sheaves in one of the ringed topoi associated to $\mathscr{X}$ :

$$
\mathscr{X}_{\text {Zar }}, \mathscr{X}_{\text {fppf }}, \mathscr{X}_{\text {ÉT }}, \mathscr{X}_{\text {lis-ét }}, \mathscr{X}_{\text {ét }} \text {. }
$$

The first three are all meant to be big topoi while the fourth is developed in [LM00], but note that there are errors in their treatment, which have been fixed by Olsson in [Ols07]. The fifth topos is the small étale topos: generated by étale morphisms from schemes $U \rightarrow \mathscr{X}$. Unless $\mathscr{X}$ is a Deligne-Mumford stack, this is an unreasonable (mostly empty) choice. We leave the proof of the following lemma to the reader (with help from the references).

Lemma A.1. There are natural equivalences of abelian categories of quasi-coherent sheaves on $\mathscr{X}_{\text {Zar }}$, $\mathscr{X}_{\text {fppf }}, \mathscr{X}_{\text {ÉT }}$, and $\mathscr{X}_{\text {lis-ét. }}$. When $\mathscr{X}$ is Deligne-Mumford, this equivalence extends to $\mathscr{X}_{\text {ét }}$.

The derived category $\mathbf{D}(\mathrm{Q} \operatorname{Coh}(\mathscr{X}))$ has the same formal properties when $\mathscr{X}$ is a stack as when it is a scheme. In particular, if $\mathscr{X}$ is regular of everywhere bounded homological dimension, any coherent sheaf defines a perfect complex in $\mathbf{D}^{\text {parf }}(\mathrm{QCoh}(\mathscr{X})) \subset \mathbf{D}(\mathrm{QCoh}(\mathscr{X}))$. (Even when $\mathscr{X}$ is equidimensional and quasi-compact, it is no longer true that the homological dimension is related to the intrinsic dimension of $\mathscr{X}$, which can be negative; it is, however, certainly bounded above by the dimension of a smooth cover of $\mathscr{X}$.)

Remark A.2. Just as in the case of a scheme, the natural functor $\mathrm{QCoh}(\mathscr{X}) \rightarrow \mathscr{M}$ od $(\mathscr{X})$ is not an exact functor of abelian categories when working with the big topologies, and therefore in the big étale and fppf topologies it is not reasonable to think about $\mathbf{D}_{\mathrm{QCoh}}\left(\mathscr{X}_{\text {fppf }}\right)$ or $\mathbf{D}_{\mathrm{QCoh}}\left(\mathscr{X}_{\text {ÉT }}\right)$. It does make sense in the lisse-étale topology, but the entire setup requires great care in that context, as the formation of the lisse-étale topos is not functorial. This is developed carefully and completely in [Ols07]. For Deligne-Mumford stacks one can work in the small étale topos $\mathscr{X}_{\text {ét }}$ and everything works just as for algebraic spaces.

We recall the following theorem, essentially due to Knudsen and Mumford [KM76] (to which the reader is referred for further details). Write $\mathrm{C}^{\text {parf }}(\mathscr{X})$ for the abelian category of perfect complexes of coherent sheaves and $\mathrm{C}_{\cong}^{\text {parf }}(\mathscr{X})$ for the subcategory in which the morphisms are precisely the quasi-isomorphisms of perfect complexes. Write Pic $\cong(\mathscr{X})$ for the Picard groupoid (the category of invertible sheaves along with isomorphisms between them on $\mathscr{X}$-schemes).

THEOREM A.3. There is a natural additive and exact functor

$$
\text { det }: \mathrm{C}_{\cong}^{\text {parf }}(\mathscr{X}) \rightarrow \operatorname{Pic} \cong(\mathscr{X})
$$

which for every locally free sheaf $\mathscr{V}$ on $\mathscr{X}$, viewed as a complex with $\mathscr{V}$ in degree 0 , takes the value $\bigwedge^{\mathrm{rk} \mathscr{V}} \mathscr{V}$

The reader should refer to [KM76, p. 23ff] for the precise properties the functor is assumed to have. For our purposes, it suffices to note that one can compute the determinant of a coherent sheaf $\mathscr{F}$ on a regular stack by taking a locally free resolution $\mathscr{V} \bullet \rightarrow \mathscr{F}$ (when one exists) and tensoring the top wedge powers of the $\mathscr{V}^{i}$ with alternating signs. 


\section{TWISTED SHEAVES AND THE PERIOD-INDEX PROBLEM}

Definition A.4. Given a coherent sheaf $\mathscr{F}$ on $\mathscr{X}$ whose image in $\mathbf{D}(\mathrm{QCoh}(\mathscr{X}))$ is perfect, the determinant of $\mathscr{F}$ is $\operatorname{det} \mathscr{F}$.

Proposition A.5. Let $\mathscr{X}$ be a regular Artin stack and $\mathscr{C} \subset \mathscr{X}$ an integral effective Cartier divisor. A coherent sheaf $\mathscr{F}$ on $\mathscr{C}$ of generic rank $r$ has determinant $\mathscr{O}_{\mathscr{X}}(r \mathscr{C})$ (as a perfect complex on $\mathscr{X}$ ).

Proof. We would like to thank Moret-Bailly for pointing out a simplification of our original proof. Let $\mathscr{U}=\mathscr{X} \backslash \mathscr{C}$ be the (open) complement of $\mathscr{C}$. Since $\left.\mathscr{F}\right|_{\mathscr{U}}=0$, there is an isomorphism $\sigma:\left.\mathscr{O}_{\mathscr{U}} \stackrel{\sim}{\rightarrow} \operatorname{det} \mathscr{F}\right|_{\mathscr{U}}$. To show that $\sigma$ extends to an isomorphism $\mathscr{O}_{\mathscr{X}}(r \mathscr{C}) \stackrel{\sim}{\rightarrow} \operatorname{det} \mathscr{F}$, it suffices to show this locally in the smooth topology of $\mathscr{X}$, as the descent datum on $\sigma$ will descend the result back to $\mathscr{X}$. Thus, it suffices to prove the result when $\mathscr{X}$ is a scheme. To prove it in this case, note that an extension must exist for some $r$ by standard properties of invertible sheaves. To determine $r$, it thus suffices to prove the result when $\mathscr{X}$ is the spectrum of a discrete valuation ring and $\mathscr{C}$ is the closed point, where the result is immediate.

Let $\mathscr{X}$ be a regular Artin stack and $\iota: \mathscr{C} \hookrightarrow \mathscr{X}$ an integral effective Cartier divisor. Suppose that we are given a coherent sheaf $\mathscr{F}$ on $\mathscr{X}$ and a coherent quotient of the restriction $\rho:\left.\mathscr{F}\right|_{\mathscr{C}} \rightarrow \mathscr{Q}$.

Definition A.6. With the above notation, the elementary transform of $\mathscr{F}$ along $\rho$ is

$$
\operatorname{ker}\left(\mathscr{F} \rightarrow \iota_{*} \mathscr{F}_{\mathscr{C}} \rightarrow \iota_{*} \mathscr{Q}\right) .
$$

Corollary A.7. Given a coherent sheaf $\mathscr{F}$ on $\mathscr{X}$ and a quotient $\rho: \mathscr{F}_{\mathscr{C}} \rightarrow \mathscr{Q}$, the determinant of the elementary transform of $\mathscr{F}$ along $\rho$ is $\operatorname{det}(\mathscr{F}) \otimes \mathscr{O}\left(-\left(\operatorname{rk}_{\mathscr{C}} \mathscr{Q}\right) \mathscr{C}\right)$.

The corollary applies, for example, if $\mathscr{X}$ is quasi-compact and regular.

\section{ACKnowledgements}

This paper (along with [Lie07b] and [Lie07a]) is based upon part of my $\mathrm{PhD}$ thesis, written under the guidance of Aise Johan de Jong [Lie04]. I also received numerous helpful comments from Jean-Louis Colliot-Thélène, Laurent Moret-Bailly, Martin Olsson, David Saltman, Jason Starr, Burt Totaro, and Olivier Wittenberg. Finally, I owe a great debt of gratitude to the referee, who gave this paper a very careful reading and helped to drastically improve the exposition. As usual, any remaining errors are entirely my own.

\section{REFERENCES}

Cal00 A. Căldăraru, Derived categories of twisted sheaves on Calabi-Yau manifolds, Thesis, Cornell University (2000).

Art71 M. Artin, On the joins of Hensel rings, Adv. Math. 7 (1971), 282-296.

Art82 M. Artin, Local structure of maximal orders on surfaces, in Brauer groups in ring theory and algebraic geometry (Wilrijk, 1981), Lecture Notes in Mathematics, vol. 917 (Springer, Berlin, 1982), 146-181.

AD03 M. Artin and A. J. de Jong, Stable orders over surfaces, Preprint (2003).

AM72 M. Artin and D. Mumford, Some elementary examples of unirational varieties which are not rational, Proc. London Math. Soc. (3) 25 (1972), 75-95.

Bas68 H. Bass, Algebraic K-theory (W. A. Benjamin, New York, 1968).

Bou89 N. Bourbaki. Commutative algebra (Springer, New York, 1989), Chapters 1-7.

Col02 J.-L. Colliot-Thélène, Exposant et indice d'algèbres simples centrales non ramifiées, Enseign. Math. (2) 48 (2002), 127-146. 


\section{LIEBLICH}

Dej03 A. J. de Jong, A result of Gabber, Preprint (2003). www.math.columbia.edu/ dejong/papers/2-gabber.pdf.

Dej04 A. J. de Jong, The period-index problem for the Brauer group of an algebraic surface, Duke Math. J. 123 (2004), 71-94.

DS03 A. J. de Jong and J. Starr, Every rationally connected variety over the function field of a curve has a rational point, Amer. J. Math. 125 (2003), 567-580.

EHKV01 D. Edidin, B. Hassett, A. Kresch and A. Vistoli, Brauer groups and quotient stacks, Amer. J. Math. 123 (2001), 761-777.

FD93 B. Farb and R. K. Dennis, Noncommutative algebra, Graduate Texts in Mathematics, vol. 144 (Springer, New York, 1993).

Gab81 O. Gabber, Some theorems on Azumaya algebras, in The Brauer group (Seminar, Les Plans-surBex, 1980), Lecture Notes in Mathematics, vol. 844 (Springer, Berlin, 1981), 129-209.

Gir71 J. Giraud, Cohomologie non abélienne. Die Grundlehren der mathematischen Wissenschaften, Band 179 (Springer, Berlin, 1971).

GHS03 T. Graber, J. Harris and J. Starr, Families of rationally connected varieties, J. Amer. Math. Soc. 16 (2003), 57-67.

Gro68a A. Grothendieck, Le groupe de Brauer. II. Théorie cohomologique, in Dix Exposés sur la Cohomologie des Schémas (North-Holland, Amsterdam, 1968), 67-87.

Gro68b A. Grothendieck, Le groupe de Brauer. III. Exemples et compléments, in Dix Exposés sur la Cohomologie des Schémas (North-Holland, Amsterdam, 1968), 88-188.

SGA1 A. Grothendieck, Revêtements étales et groupe fondamental, Séminaire de Géométrie Algébrique du Bois Marie 1960-1961 (SGA 1). With two papers by M. Raynaud, Lecture Notes in Mathematics, vol. 224 (Springer, Berlin, 1971).

GY98 V. I. Guletskiı̌ and V. I. Yanchevskiŭ, Torsion of the Brauer groups of curves defined over multidimensional local fields, Dokl. Nats. Akad. Nauk Belarusi, 42 (1998), 5-8, 121.

Hoo82 R. T. Hoobler, When is $\operatorname{Br}(X)=\operatorname{Br}^{\prime}(X)$ ?, in Brauer groups in ring theory and algebraic geometry (Wilrijk, 1981), Lecture Notes in Mathematics, vol. 917 (Springer, Berlin, 1982), 231-244.

HL97 D. Huybrechts and M. Lehn, The geometry of moduli spaces of sheaves, Aspects of Mathematics, vol. E31 (Vieweg, Braunschweig, 1997).

KS99 A. King and A. Schofield, Rationality of moduli of vector bundles on curves, Indag. Math. (N.S.) 10 (1999), 519-535.

KM76 F. F. Knudsen and D. Mumford, The projectivity of the moduli space of stable curves. I. Preliminaries on "det" and "Div", Math. Scand. 39 (1976), 19-55.

KP95 A. Kouvidakis and T. Pantev, The automorphism group of the moduli space of semistable vector bundles, Math. Ann. 302 (1995), 225-268.

Kre03 A. Kresch, Hodge-theoretic obstruction to the existence of quaternion algebras, Bull. London Math. Soc. 35 (2003), 109-116.

T58 S. Lang and J. Tate, Principal homogeneous spaces over abelian varieties, Amer. J. Math. 80 (1958), 659-684.

LW54 S. Lang and A. Weil, Number of points of varieties in finite fields, Amer. J. Math. 76 (1954), 819-827.

Lan06 A. Langer, Moduli spaces and Castelnuovo-Mumford regularity of sheaves on surfaces, Amer. J. Math. 128 (2006), 373-417.

LM00 G. Laumon and L. Moret-Bailly, Champs algébriques, Ergebnisse der Mathematik und ihrer Grenzgebiete (3). A Series of Modern Surveys in Mathematics vol. 39 (Springer, Berlin, 2000).

Lic68 S. Lichtenbaum, The period-index problem for elliptic curves, Amer. J. Math. 90 (1968), 1209-1223.

Lie04 M. Lieblich, Moduli of twisted sheaves and generalized Azumaya algebras, PhD thesis, Massachusetts Institute of Technology (2004). 


\section{TWISTED SHEAVES AND THE PERIOD-INDEX PROBLEM}

Lie06 M. Lieblich. Period and index in the Brauer group of an arithmetic surface (with an appendix by Daniel Krashen), Preprint (2006), arXiv:0706.1072.

Lie07a M. Lieblich, Compactified moduli of projective bundles, Preprint (2007), arXiv:0706.1311.

Lie07b M. Lieblich, Moduli of twisted sheaves, Duke Math. J. 138 (2007), 23-118.

MFK94 D. Mumford, J. Fogarty and F. Kirwan. Geometric invariant theory, third edition, Ergebnisse der Mathematik und ihrer Grenzgebiete (2) (Springer, Berlin, 1994).

Nak35 T. Nakayama, Über die direkte Zerlegung einer Divisionsalgebra, Japan. J. Math. 12 (1935), 65-70.

Gra96 K. G. O'Grady. Moduli of vector bundles on projective surfaces: some basic results, Invent. Math. 123 (1996), 141-207.

Ols07 M. Olsson, Sheaves on Artin stacks, J. reine angew. Math. 603 (2007), 55-112.

Ram73 S. Ramanan, The moduli spaces of vector bundles over an algebraic curve, Math. Ann. 200 (1973), 69-84.

Ray70 M. Raynaud, Anneaux locaux henséliens, Lecture Notes in Mathematics, vol. 169 (Springer, Berlin, 1970).

Sal97 D. J. Saltman, Division algebras over p-adic curves, J. Ramanujan Math. Soc. 12 (1997), 25-47.

Sal98 D. J. Saltman, Correction to: "Division algebras over p-adic curves", J. Ramanujan Math. Soc. 13 (1998), 125-129.

Ser95 J.-P. Serre, Local Fields, Graduate Texts in Mathematics, vol. 67 (Springer, New York, 1995).

Sil92 J. H. Silverman, The arithmetic of elliptic curves, Graduate Texts in Mathematics, vol. 106 (Springer, New York, 1992). (Corrected reprint of the 1986 original).

Van86 M. Van den Bergh, The algebraic index of a division algebra, in Ring theory (Antwerp, 1985), Lecture Notes in Mathematics, vol. 1197 (Springer, Berlin, 1986), 190-206.

Vis89 A. Vistoli, Intersection theory on algebraic stacks and on their moduli spaces, Invent. Math. 97 (1989), 613-670.

Wat79 W. C. Waterhouse, Introduction to affine group schemes, Graduate Texts in Mathematics, vol. 66 (Springer, New York, 1979).

Yos03 K. Yoshioka, Twisted stability and the Fourier-Mukai transform, I, Compositio Math. 138 (2003), $261-288$.

Max Lieblich lieblich@math.princeton.edu

Department of Mathematics, Fine Hall, Washington Road, Princeton NJ 08544-1000, USA 